\title{
Une formule pour les extensions de foncteurs composés
}

\author{
par
}

Alain Troesch (Paris)

\begin{abstract}
Let $p$ be a prime, and let $\mathcal{F}$ be the category of functors from the finite $\mathbb{F}_{p}$-vector spaces to all $\mathbb{F}_{p}$-vector spaces. The object Id of $\mathcal{F}$ is the inclusion functor. Let $F$ and $G$ be two objects in $\mathcal{F}$. If $F$ and $G$ satisfy suitable conditions, the main result of this paper allows one to compute $\operatorname{Ext}_{\mathcal{F}}^{*}(\mathrm{Id}, G \circ F)$ from the knowledge of $\operatorname{Ext}_{\mathcal{F}}^{*}(\operatorname{Id}, F)$ and $\operatorname{Ext}_{\mathcal{F}}^{*}(\operatorname{Id}, G)$.
\end{abstract}

Introduction. Soit $p$ un nombre premier. Soit $\mathcal{P}$ la catégorie des foncteurs strictement polynomiaux introduite par Friedlander et Suslin [6]. Il s'agit d'une catégorie dont les objets sont définis de manière similaire aux endofoncteurs de la catégorie des espaces vectoriels de dimension finie sur le corps $\mathbb{F}_{p}$, hormis le fait qu'on impose, pour un objet $P$, et pour tout couple d'espaces vectoriels $(V, W)$, que l'application $P_{V, W}$ définissant l'action sur les flèches soit (formellement) polynomiale. $\mathrm{Si}$, pour un objet $P$, toutes les applications $P_{V, W}$ sont des polynômes homogènes de degré $d$, on dira de surcroît que $P$ est homogène de degré $d$. La catégorie $\mathcal{P}$ est la somme directe de ses sous-catégories pleines $\mathcal{P}_{d}$ constituées des foncteurs homogènes de degré $d$.

Les puissances symétriques $S^{n}$, extérieures $\Lambda^{n}$ et divisées $\Gamma^{n}$ sont des exemples d'objets de $\mathcal{P}_{n}$. Un autre exemple important de foncteur strictement polynomial est le twist de Frobenius Tw consistant à prendre l'identité sur les objets, et la puissance $p$-ième formelle sur les morphismes. En composant $n$ fois à droite un foncteur $F$ de $\mathcal{P}$ par le twist Tw, on obtient un foncteur noté $F^{(n)}$, dont le degré est $p^{n}$ fois plus grand que celui de $F$.

La catégorie $\mathcal{P}$ a suffisamment d'injectifs : on peut y faire de l'algèbre homologique. Par exemple, Friedlander et Suslin [6] ont calculé les espaces vectoriels $\operatorname{Ext}_{\mathcal{F}}^{*}\left(\operatorname{Id}^{(n)}, S^{p^{n}}\right)$, adaptant des résultats antérieurs de Franjou, Lannes et Schwartz [5] obtenus dans la catégorie $\mathcal{F}$ des foncteurs (au sens

2000 Mathematics Subject Classification: 18G05, 18G10, 18G15, 18G40, 55S10.

L'auteur a bénéficié pendant une partie de la réalisation de ce travail d'une bourse doctorale Marie Curie de la Commission Européenne HPMT-CT-2000-00075. 
usuel) des espaces vectoriels finis vers tous les espaces vectoriels sur $\mathbb{F}_{p}$. Ces calculs ont permis de démontrer un résultat de finitude de la cohomologie rationnelle des schémas en groupes.

Via la catégorie $\mathcal{F}$, la catégorie $\mathcal{P}$ est aussi étroitement liée à la catégorie $\mathcal{U}$ des modules instables sur l'algèbre de Steenrod [7, 13].

L'auteur a poursuivi dans la voie des calculs de Franjou, Lannes et Schwartz, et de Friedlander et Suslin, en déterminant l'espace vectoriel $\operatorname{Ext}_{\mathcal{P}}^{*}\left(\operatorname{Id}^{(h+k)}, S^{p^{k}} \circ S^{p^{h}}\right)$ dans le cas où $p=2$ (cf. $\left.[14,15,16]\right)$. Il obtient la structure de module de Yoneda de ces objets, c'est-à-dire la structure de module à droite sur l'algèbre $\operatorname{Ext}_{\mathcal{P}}^{*}\left(\mathrm{Id}^{(h+k)}, \mathrm{Id}^{(h+k)}\right)$, les produits interne pour l'algèbre et externe pour le module étant donnés par la composition de Yoneda des extensions.

Dans la suite de ces calculs, on propose ici une formule générale, modulo quelques hypothèses, pour $\operatorname{Ext}_{\mathcal{F}}^{*}(\operatorname{Id}, G \circ F)$ en fonction de $\operatorname{Ext}_{\mathcal{F}}^{*}(\operatorname{Id}, G)$, de $\operatorname{Ext}_{\mathcal{F}}^{*}(\mathrm{Id}, F)$ et des modules $\operatorname{Ext}_{\mathcal{P}}^{*}\left(\operatorname{Id}^{(h+k)}, S^{p^{k}} \circ S^{p^{h}}\right)$. On notera que ces derniers ne sont connus que si $p=2$.

ThÉORÈme A (Théorèmes 5.1 et 5.2). Soient $F$ et $G$ deux objets de $\mathcal{P}$, homogènes de degrés respectifs $p^{h}$ et $p^{k}$. On suppose que $\operatorname{Ext}_{\mathcal{P}}^{*}\left(\operatorname{Id}^{(h)}, F\right)$ et $\operatorname{Ext}_{\mathcal{P}}^{*}\left(\operatorname{Id}^{(k)}, G\right)$ ont une structure de module de Yoneda triviale. Alors, on a un isomorphisme gradué de modules de Yoneda

$$
\begin{aligned}
& \operatorname{Ext}_{\mathcal{P}}^{*}\left(\operatorname{Id}^{(h+k)}, G \circ F\right) \\
& \quad \cong \operatorname{Ext}_{\mathcal{P}}^{*}\left(\operatorname{Id}^{(h)}, F\right) \otimes \operatorname{Ext}_{\mathcal{P}}^{*}\left(\operatorname{Id}^{(k)}, G\right) \otimes \operatorname{Ext}_{\mathcal{P}}^{*}\left(\operatorname{Id}^{(h+k)}, S^{p^{k}} \circ S^{p^{h}}\right) .
\end{aligned}
$$

La structure de module de Yoneda du produit tensoriel provient de la structure de module du troisième facteur.

En application, on montre comment ce théorème permet de contourner une bonne partie des calculs de [16] pour déterminer $\operatorname{Ext}_{\mathcal{P}}^{*}\left(\operatorname{Id}^{(h+k)}, S^{p^{k}} \circ S^{p^{h}}\right)$, dans le cas où $p=2$, et obtenir plus généralement le

ThÉorème B. La série de Poincaré $\varphi_{h_{1}, \ldots, h_{l}}$ de l'espace vectoriel gradué $\operatorname{Ext}_{\mathcal{P}}^{*}\left(\operatorname{Id}^{\left(h_{1}+\ldots+h_{l}\right)}, S^{2^{h_{l}}} \circ \ldots \circ S^{2^{h_{1}}}\right)$ est donnée par

$$
\varphi_{h_{1}, \ldots, h_{l}}(t)=\frac{\prod_{i=1}^{h_{1}+\ldots+h_{l}}\left(1-t^{2^{i}-1}\right)}{\prod_{i=1}^{h_{1}}\left(1-t^{2^{i}-1}\right) \ldots \prod_{i=1}^{h_{l}}\left(1-t^{2^{i}-1}\right)} .
$$

Sa structure de module de Yoneda est triviale.

Dans cette introduction, et dans la suite de cet article, nous présentons délibérément tous les résultats dans le cadre de la catégorie $\mathcal{P}$. Nous pointons le doigt sur le fait que les résultats obtenus dans la catégorie $\mathcal{P}$ peuvent se transcrire dans la catégorie $\mathcal{F}$ utilisée par Franjou, Lannes et Schwartz [5]. En effet, il existe des théorèmes de comparaison des groupes d'extensions dans chacune de ces catégories [4]. Ces résultats ne donnent pas une cor- 
respondance complète; néanmoins, dans le cas où la première variable est Id, la comparaison des résultats de Franjou, Lannes et Schwartz dans la catégorie $\mathcal{F}$ et de ceux de Friedlander et Suslin dans la catégorie $\mathcal{P}$ permet de donner une correspondance complète entre les modules d'extensions dans les catégories $\mathcal{F}$ et $\mathcal{P}[14,17]$.

1. Catégories de foncteurs - Rappels. Nous rappelons brièvement quelques faits concernant la catégorie $\mathcal{P}$ des foncteurs strictement polynomiaux. Nous suivons l'approche de Friedlander et Suslin [6].

Quelques définitions. Soit $p$ un entier premier quelconque. Soit $\mathcal{E}$ la catégorie des espaces vectoriels sur $\mathbb{F}_{p}$, et $\mathcal{E}^{f}$ sa sous-catégorie pleine constituée des espaces de dimension finie.

Soit $(V, W)$ un couple d'objets de $\mathcal{E}^{f}$. Un morphisme strictement polynomial de $V$ dans $W$ est un élément de $S^{*}\left(V^{*}\right) \otimes W$. L'espace des morphismes strictement polynomiaux de $V$ vers $W$ est noté $\operatorname{Hom}_{\text {pol }}(V, W)$. Un morphisme strictement polynomial de $V$ vers $W$ est homogène de degré $d$ s'il est élément de $S^{d}\left(V^{*}\right) \otimes W$.

Un foncteur strictement polynomial $P$ est la donnée d'une application $V \mapsto P(V)$ des objets de $\mathcal{E}^{f}$ vers les objets de $\mathcal{E}^{f}$, et d'une application qui à tout couple $(V, W)$ dans $\mathcal{E}^{f}$ associe un morphisme strictement polynomial $P_{V, W}$ de $\operatorname{Hom}(V, W)$ vers $\operatorname{Hom}(P(V), P(W))$, telles que les conditions usuelles requises pour un foncteur (compatibilité avec l'identité et avec la composition) soient satisfaites. On dit qu'un foncteur strictement polynomial est homogène de degré $d$ si tous les morphismes strictement polynomiaux $P_{V, W}$ sont homogènes de degré $d$.

On désigne par $\mathcal{P}$ la catégorie dont les objets sont les foncteurs strictement polynomiaux, et les morphismes sont les transformations naturelles dans le sens usuel. La sous-catégorie pleine de $\mathcal{P}$ constituée des foncteurs homogènes de degré $d$ est notée $\mathcal{P}_{d}$.

Les puissances symétriques $S^{n}$, extérieures $\Lambda^{n}$ et divisées $\Gamma^{n}$ sont des objets de $\mathcal{P}_{n}$. Un autre exemple important est le twist de Frobenius $\mathrm{Tw} \in \mathcal{P}_{p}$. Ce foncteur consiste à prendre l'identité sur les objets, et la puissance $p$-ième formelle sur les morphismes. Le twist de Frobenius $F^{(1)}$ d'un foncteur $F \in \mathcal{P}$ est alors défini par la composition $F \circ \mathrm{Tw}$. Plus généralement son $n$-ième twist de Frobenius $F^{(n)}$ est obtenu en itérant $n$ fois ce processus.

On décrit maintenant un système de cogénérateurs injectifs de $\mathcal{P}$.

Proposition 1.1 (Friedlander-Suslin [6]). Les foncteurs $S^{i_{1}} \otimes \ldots \otimes S^{i_{s}}$, $i_{1}+\ldots+i_{s}=d$, forment un système de cogénérateurs injectifs de $\mathcal{P}_{d}$.

DÉfinition 1.2. Soit $F$ un foncteur dans $\mathcal{P}_{d}$. Une $S$-résolution de $F$ dans $\mathcal{P}$ est une résolution injective de $F$ construite à partir des cogénérateurs injectifs de la proposition 1.1. 
En d'autres termes, une $S$-résolution d'un foncteur $F$ de $\mathcal{P}_{d}$ est une résolution dont tous les termes sont des sommes directes de produits tensoriels de puissances symétriques de degré total $d$. Une telle résolution existe toujours, mais n'est pas unique.

Extensions dans la catégorie $\mathcal{P}$. Friedlander et Suslin [6] ont été les premiers à s'intéresser à des calculs d'extensions dans la catégorie $\mathcal{P}$. Leur motivation était l'obtention d'un résultat de finitude de la cohomologie rationnelle des schémas en groupes. Ils obtiennent :

ThÉORÈME 1.3 (Friedlander-Suslin [6]). Il existe une famille $e_{l}^{(n-l)}$ d'éléments de $\operatorname{Ext}_{\mathcal{P}}^{2 p^{l-1}}\left(\mathrm{Id}^{(n)}, \mathrm{Id}^{(n)}\right), 0<l \leq n$, telle que l'algèbre de Yoneda $\operatorname{Ext}_{\mathcal{P}}^{*}\left(\operatorname{Id}^{(n)}, \mathrm{Id}^{(n)}\right)$ est le quotient de l'algèbre commutative engendrée par la famille $\left(e_{l}^{(n-l)}\right)_{0<l \leq n}$ par les relations $\left(e_{l}^{(n-l)}\right)^{p}=0$. Soit $h$ tel que $0 \leq h \leq n$. Le module de Yoneda à droite $\operatorname{Ext}_{\mathcal{P}}^{*}\left(\operatorname{Id}^{(n)}, S^{p^{h}(n-h)}\right)$ est le quotient de l'algèbre de Yoneda $\operatorname{Ext}_{\mathcal{P}}^{*}\left(\operatorname{Id}^{(n)}, \operatorname{Id}^{(n)}\right)$ par l'idéal engendré par $e_{1}^{(n-1)}, \ldots, e_{h}^{(n-h)}$. En particulier, $\operatorname{Ext}_{\mathcal{P}}^{*}\left(\operatorname{Id}^{(n)}, S^{p^{h}(n-h)}\right)$ hérite une structure d'algèbre.

Ce résultat est à rapprocher du résultat antérieur similaire obtenu par Franjou, Lannes et Schwartz [5] dans la catégorie $\mathcal{F}$ des foncteurs de $\mathcal{E}^{f}$ vers $\mathcal{E}$. La méthode utilisée est identique à celle de Franjou, Lannes et Schwartz. Elle repose en particulier sur un théorème d'annulation dû à Pirashvili :

ThÉorème 1.4 (Pirashvili [10]). Soient $F$ et $G$ deux objets de $\mathcal{P}$ sans terme constant (c'est-à-dire $F(0)=0=G(0)$ ). Soit $A$ un foncteur additif, par exemple $A=\mathrm{Id}^{(n)}$. Alors $\operatorname{Ext}_{\mathcal{P}}^{*}(A, G \otimes F)=0$.

Une version plus générale de ce théorème $[1,11]$ permet d'obtenir :

ThÉORÈme 1.5. Soient $F, G$ et $H$ des foncteurs strictement polynomiaux sans terme constant, et $A$ un foncteur additif. Alors $\operatorname{Ext}_{\mathcal{P}}^{*}(A, F \circ$ $(G \otimes H))=0$.

Défaut d'exactitude de la composition à gauche. Pour obtenir des extensions de foncteurs composés, on sera amené, comme dans [15, 14, 16], à composer des complexes à gauche ou à droite par un foncteur. Il est intéressant ce faisant de ne pas perdre les informations concernant la cohomologie. La composition à droite est exacte; en revanche, la composition à gauche ne l'est pas. Dans $[15,14,16]$, l'auteur contourne ce problème en montrant le théorème ci-dessous.

THÉORÈme 1.6 (cf. [15]). Soient $P$ un foncteur strictement polynomial dans $\mathcal{P}_{p^{k}}$, et $\mathcal{C}^{\bullet}$ un complexe d'objets de $\mathcal{P}_{p^{h}}$ sans terme constant. Alors $\forall n \geq 0, \quad \operatorname{Ext}_{\mathcal{P}}^{*}\left(\operatorname{Id}^{(h+k)}, \mathrm{H}^{n}\left(P\left(\mathcal{C}^{\bullet}\right)\right)\right)=\operatorname{Ext}_{\mathcal{P}}^{*}\left(\operatorname{Id}^{(h+k)}, P\left(\mathrm{H}^{n}\left(\mathcal{C}^{\bullet}\right)\right)\right)$. 
Ce théorème affirme que, modulo les extensions de première variable l'identité, on peut considérer que la composition à gauche est exacte.

Lien avec la catégorie $\mathcal{F}$. Enfin, on rappelle comment déduire des modules d'extensions dans la catégorie $\mathcal{P}$ les modules d'extensions similaires dans la catégorie $\mathcal{F}$.

ThÉORÈme 1.7 (cf. $[15,17])$. Soit $F \in \mathcal{P}_{p^{h}}$. L'espace $\operatorname{Ext}_{\mathcal{P}}^{s}\left(\operatorname{Id}^{(h)}, F\right)$ est nul si $s>2 p^{h}-2$. De plus, on a des isomorphismes de modules de Yoneda

$$
\begin{aligned}
\operatorname{Ext}_{\mathcal{P}}^{*}\left(\operatorname{Id}^{(h+k)}, F^{(k)}\right) & =\operatorname{Ext}_{\mathcal{P}}^{*}\left(\operatorname{Id}^{(h)}, F\right) \otimes \operatorname{Ext}_{\mathcal{P}}^{*}\left(\operatorname{Id}^{(h+k)}, S^{p^{h}(k)}\right), \\
\operatorname{Ext}_{\mathcal{F}}^{*}(\operatorname{Id}, F) & =\operatorname{Ext}_{\mathcal{P}}^{*}\left(\operatorname{Id}^{(h)}, F\right) \otimes \operatorname{Ext}_{\mathcal{F}}^{*}\left(\operatorname{Id}, S^{p^{h}}\right) .
\end{aligned}
$$

La structure de module du produit tensoriel est le produit tensoriel usuel de deux modules sur deux algèbres $A$ et $A^{\prime}$ (cf. [2]). On remarquera que le produit tensoriel $\operatorname{Ext}_{\mathcal{P}}^{*}\left(\operatorname{Id}^{(h)}, \operatorname{Id}^{(h)}\right) \otimes_{\mathbb{F}_{p}} \operatorname{Ext}_{\mathcal{P}}^{*}\left(\operatorname{Id}^{(h+k)}, S^{p^{h}(k)}\right)$ est isomorphe en tant qu'algèbre à $\operatorname{Ext}_{\mathcal{P}}^{*}\left(\operatorname{Id}^{(h+k)}, \operatorname{Id}^{(h+k)}\right)$.

2. $S$-résolutions réduites. Dans cette partie, on construit des $S$-résolutions ayant un nombre minimal de facteurs $S^{p^{k}}$. Le seul point original est la nullité de $\operatorname{Ext}_{\mathcal{P}}^{1}\left(\operatorname{Id}^{(k)}, \operatorname{Id}^{(k)}\right)$.

DÉfinition 2.1. Soit $G$ un foncteur strictement polynomial homogène de degré $p^{k}$. On appellera $S$-résolution Id-réduite, ou simplement réduite, une $S$-résolution $\mathcal{R}_{G}^{\bullet}$ telle que le nombre de facteurs $S^{p^{k}}$ dans $\mathcal{R}_{G}^{s}$ soit exactement égal à la dimension de $\operatorname{Ext}^{s}\left(\operatorname{Id}^{(k)}, G\right)$.

On ne peut pas faire mieux, à cause du théorème de Pirashvili.

Une définition équivalente en termes de suites spectrales. Soient $\mathcal{C}^{\bullet}$ un complexe d'objets de $\mathcal{P}$, et $T \in \mathcal{P}$. On rappelle $[3,18]$ qu'on définit les première et seconde suites spectrales d'hypercohomologie $\mathbf{I}\left(T, \mathcal{C}^{\bullet}\right)$ et $\mathbf{I I}\left(T, \mathcal{C}^{\bullet}\right)$ de $\mathcal{C}^{\bullet}$ relativement à $T$ en considérant les filtrations verticale et horizontale du bicomplexe $\operatorname{Hom}_{\mathcal{P}}\left(T, I^{\bullet \bullet}\right), I^{\bullet \bullet}$ étant une résolution injective de CartanEilenberg [3] du complexe $\mathcal{C}^{\bullet}$. Elles convergent vers des gradués d'un même espace, et sont données en petits rangs par

$$
\mathbf{I}_{1}^{s, t}\left(T, \mathcal{C}^{\bullet}\right)=\operatorname{Ext}_{\mathcal{P}}^{t}\left(T, \mathcal{C}^{s}\right), \quad \mathbf{I I}_{2}^{s, t}\left(T, \mathcal{C}^{\bullet}\right)=\operatorname{Ext}_{\mathcal{P}}^{s}\left(T, \mathrm{H}^{t}\left(\mathcal{C}^{\bullet}\right)\right) .
$$

Dans les conventions d'indexation utilisées ici, les différentielles de rang $r$ sont de bidegré $(r, 1-r)$.

Étant donné une $S$-résolution $\mathcal{R}_{G}^{\bullet}$ d'un foncteur $G$, la suite spectrale $\mathbf{I}\left(\operatorname{Id}^{(k)}, \mathcal{R}_{G}^{\bullet}\right)$ est nulle ailleurs que sur sa ligne d'ordonnée 0 , car $\mathcal{R}_{G}^{\bullet}$ est une résolution injective. Ainsi, elle dégénère au rang 2 . Grâce au théorème d'annulation de Pirashvili et au théorème 1.3, on obtient : 
Proposition 2.2. La S-résolution $\mathcal{R}_{G}^{\bullet}$ est réduite si et seulement si la suite spectrale $\mathbf{I}\left(\mathrm{Id}^{(k)}, \mathcal{R}_{G}^{\bullet}\right)$ dégénère au rang 1 .

Existence d'une S-résolution réduite. L'objet des lemmes 2.3-2.5 est de montrer que tout foncteur admet au moins une $S$-résolution réduite.

Lemme 2.3. Soit $G$ un objet de $\mathcal{P}_{p^{k}}$ tel que $\operatorname{dim} \operatorname{Ext}_{\mathcal{P}}^{0}\left(\operatorname{Id}^{(k)}, G\right)=0$. Alors $G$ s'injecte dans une somme de produits tensoriels non triviaux (c'està-dire non réduits à un terme) de puissances symétriques.

Démonstration. Soit $\mathcal{R}_{G}^{\bullet}$ une $S$-résolution de $G$, munie d'une décomposition de ses termes en somme directe de produits tensoriels de puissances symétriques. Seuls les facteurs directs égaux à $S^{p^{k}}$ de $\mathcal{R}_{G}^{0}$ contribuent à $\operatorname{Ext}_{\mathcal{P}}^{0}\left(\operatorname{Id}^{(k)}, G\right)$, à cause du théorème de Pirashvili. Puisque $\operatorname{Ext}_{\mathcal{P}}^{0}\left(\operatorname{Id}^{(k)}, G\right)=0$, il en résulte que la projection de l'injection $G \hookrightarrow \mathcal{R}_{G}^{0}$ sur le facteur direct de $\mathcal{R}_{G}^{0}$ obtenu en prenant la somme de tous les facteurs de la décomposition de $\mathcal{R}_{G}^{0}$ égaux à des produits tensoriels non triviaux est encore une injection. Cette injection peut constituer le point de départ d'une $S$-résolution de $G$.

Lemme 2.4. Soit $G$ un objet de $\mathcal{P}_{p^{k}}$. Il existe une injection

$$
G \hookrightarrow\left(S^{p^{k}}\right)^{\oplus n_{0}} \oplus \overline{\mathcal{R}}_{G}^{0},
$$

où $n_{0}$ est égal à la dimension de $\operatorname{Ext}_{\mathcal{P}}^{0}\left(\operatorname{Id}^{(k)}, G\right)$, et $\overline{\mathcal{R}}_{G}^{0}$ est une somme directe de produits tensoriels non triviaux de puissances symétriques.

Démonstration. La preuve relève de résultats basiques d'algèbre homologique. On utilise un ingrédient particulier à cette situation : la nullité de $\operatorname{Ext}_{\mathcal{P}}^{1}\left(\operatorname{Id}^{(k)}, \operatorname{Id}^{(k)}\right)$.

On opère par récurrence sur la dimension $n_{0} \operatorname{de} \operatorname{Ext}_{\mathcal{P}}^{0}\left(\operatorname{Id}^{(k)}, G\right)$, c'est-àdire de $\operatorname{Hom}_{\mathcal{P}}\left(\operatorname{Id}^{(k)}, G\right)$. Si $n_{0}=0$, c'est le lemme 2.3. Supposons que $n_{0}>0$. Alors, il existe un morphisme non nul Id ${ }^{(k)} \rightarrow G$. Il est injectif, car Id ${ }^{(k)}$ est simple. Soit $C$ son conoyau. On obtient une suite exacte courte

$$
0 \rightarrow \mathrm{Id}^{(k)} \rightarrow G \rightarrow C \rightarrow 0 .
$$

Puisque $\operatorname{Ext}_{\mathcal{P}}^{1}\left(\operatorname{Id}^{(k)}, \operatorname{Id}^{(k)}\right)=0$, la suite exacte longue obtenue en appliquant $\operatorname{Ext}_{\mathcal{P}}^{*}\left(\operatorname{Id}^{(k)},-\right)$ fournit une suite exacte courte

$$
0 \rightarrow \operatorname{Ext}_{\mathcal{P}}^{0}\left(\operatorname{Id}^{(k)}, \operatorname{Id}^{(k)}\right) \rightarrow \operatorname{Ext}_{\mathcal{P}}^{0}\left(\operatorname{Id}^{(k)}, G\right) \rightarrow \operatorname{Ext}_{\mathcal{P}}^{0}\left(\operatorname{Id}^{(k)}, C\right) \rightarrow 0 .
$$

La dimension de $\operatorname{Ext}_{\mathcal{P}}^{0}\left(\operatorname{Id}^{(k)}, C\right)$ est donc $n_{0}-1$. On peut appliquer l'hypothèse de récurrence à $C$. Il existe donc une injection

$$
C \hookrightarrow\left(S^{p^{k}}\right)^{\oplus\left(n_{0}-1\right)} \oplus \overline{\mathcal{R}}_{C}^{0},
$$

où $\overline{\mathcal{R}}_{C}^{0}$ est une somme directe de produits tensoriels non triviaux de puissances symétriques. De même, on a une injection $\operatorname{Id}^{(k)} \hookrightarrow S^{p^{k}}$. Par consé- 
quent, on obtient une injection

$$
G \hookrightarrow S^{p^{k}} \oplus\left(S^{p^{k}}\right)^{\oplus\left(n_{0}-1\right)} \oplus \overline{\mathcal{R}}_{C}^{0}
$$

(construction d'une résolution injective du terme central d'une suite exacte courte à partir de résolutions injectives des deux autres termes [3]).

LEMme 2.5. Tout foncteur $G$ de $\mathcal{P}_{p^{k}}$ admet au moins une résolution réduite.

Démonstration. On construit la résolution pas à pas avec le lemme 2.4, de la même manière qu'on construit une résolution injective quand il y a suffisamment d'injectifs. Les conditions de minimalité se transportent d'une étape à la suivante.

\section{S-résolutions explicites}

DÉfinition 2.6. Une $S$-résolution explicite de $G$ est la donnée d'une $S$-résolution de $G$, et d'une décomposition explicite des foncteurs $\mathcal{R}_{G}^{s}$ en somme directe de produits tensoriels de puissances symétriques.

Dans tout ce qui suit, on se fixe $G$ un foncteur homogène de degré $p^{k}$, et $\mathcal{R}_{G}^{\bullet}$ une $S$-résolution explicite réduite de $G$, munie d'une décomposition

$$
\mathcal{R}_{G}^{l}=\bigoplus_{\alpha=1}^{\alpha_{l}} S^{l, \alpha}
$$

de chacun de ses termes, où $S^{l, \alpha}$ est un produit tensoriel de puissances symétriques, de degré total $p^{k}$.

Terminologie 2.7. Un terme $S^{l, \alpha}$ apparaissant dans la décomposition (2) de $\mathcal{R}_{G}^{l}$ est appelé facteur élémentaire de degré (cohomologique) $l$ de la $S$-résolution réduite explicite $\mathcal{R}_{G}^{\bullet}$.

Graphe d'une S-résolution explicite

DÉfinition 2.8. Le graphe de la $S$-résolution explicite $\mathcal{R}_{G}^{\bullet}$ est le graphe orienté $\Gamma\left(\mathcal{R}_{G}^{\bullet}\right)$ tel que :

- les sommets sont les facteurs élémentaires de la résolution $\mathcal{R}_{G}^{\bullet}$;

- il existe une flèche du sommet $S^{l, \alpha}$ vers le sommet $S^{m, \beta}$ si et seulement si $m=l+1$ et si la composition $S^{l, \alpha} \longrightarrow \mathcal{R}_{G}^{k} \stackrel{d}{\longrightarrow} \mathcal{R}_{G}^{k+1} \longrightarrow S^{m, \beta}$ est non nulle.

DÉfinition 2.9. Soit $V$ un ensemble de sommets de $\Gamma\left(\mathcal{R}_{G}^{\bullet}\right)$. Le graphe engendré par $V$ est le plus petit sous-graphe plein $\Gamma(V)$ de $\Gamma\left(\mathcal{R}_{G}^{\bullet}\right)$ qui contient les sommets contenus dans $V$ et tel que pour tout sommet $v$ de $\Gamma(V)$ et toute arête $v \rightarrow w$ de $\Gamma\left(\mathcal{R}_{G}^{\bullet}\right), w$ est aussi un sommet de $\Gamma(V)$. 
On note $\Gamma(V, n)$ l'ensemble des sommets de degré $n$ de $\Gamma(V)$, et pour un sous-ensemble $V$ de sommets de $\Gamma\left(\mathcal{R}_{G}^{\bullet}, s\right), \mathcal{R}_{G}^{\bullet}(V)$ le complexe défini par

$$
\mathcal{R}_{G}^{t}(V)=\bigoplus_{v \in \Gamma(V, t)} v
$$

Sa différentielle est la restriction de celle de $\mathcal{R}_{G}^{\bullet}$. Par construction de $\Gamma(V, t)$, l'inclusion de chacun des termes $\mathcal{R}_{G}^{t}(V) \rightarrow \mathcal{R}_{G}^{t}$ définit un morphisme de complexes $\mathcal{R}_{G}^{\bullet}(V) \rightarrow \mathcal{R}_{G}^{\bullet}$ : le complexe $\mathcal{R}_{G}^{\bullet}(V)$ est un sous-complexe de $\mathcal{R}_{G}^{\bullet}$. Si $V=\{v\}$ est réduit à un élément, on écrira $\mathcal{R}_{G}^{\bullet}(v)$ au lieu de $\mathcal{R}_{G}^{\bullet}(\{v\})$.

3. Graphes et actions de Yoneda. On se donne $G$ un foncteur strictement polynomial homogène de degré $p^{k}$, et $\mathcal{R}_{G}^{\bullet}$ une $S$-résolution réduite explicite de $G$.

Lemme 3.1. Soit $v=S^{p^{k}}$ un facteur élémentaire en degré $s$ de la $S$ résolution explicite $\mathcal{R}_{G}^{\bullet}$. Soit $\varphi_{v}$ le morphisme $\operatorname{Id}^{(k)} \rightarrow \mathcal{R}_{G}^{s}$ tel que $\varphi_{v}$ est le morphisme de Frobenius $x^{(k)} \mapsto x^{p^{k}}$ sur le facteur $v=S^{p^{k}}$, et est nul sur les autres facteurs. Le morphisme $\varphi$ est le représentant d'une classe d'extension dans $\operatorname{Ext}_{\mathcal{P}}^{s}\left(\operatorname{Id}^{(k)}, G\right)$. De plus, l'ensemble des classes d'extensions $\left[\varphi_{v}\right]$ ainsi obtenues avec tous les facteurs élémentaires $v$ sans produit tensoriel (i.e. égaux à $\left.S^{p^{k}}\right)$ de $\mathcal{R}_{G}^{s}$ est une base de $\operatorname{Ext}_{\mathcal{P}}^{s}\left(\operatorname{Id}^{(k)}, G\right)$.

Démonstration. Ce résultat est évident du fait du théorème d'annulation de Pirashvili, et de la minimalité requise pour la $S$-résolution réduite $\mathcal{R}_{G}^{\bullet}$.

On parlera de la classe d'extension représentée par le facteur élémentaire $v=S^{p^{k}}$ pour désigner la classe représentée par le morphisme $\varphi_{v}$.

Proposition 3.2. Soient e un élément de $\operatorname{Ext}_{\mathcal{P}}^{i}\left(\operatorname{Id}^{(k)}, \operatorname{Id}^{(k)}\right)$ et $v=S^{p^{k}}$ un facteur élémentaire d'une $S$-résolution réduite explicite $\mathcal{R}_{G}^{\bullet}$ de $G$, représentant une extension $f \in \operatorname{Ext}_{\mathcal{P}}^{s}\left(\operatorname{Id}^{(k)}, G\right)$. Si les sommets de $\Gamma(\{v\}, s+i)$ sont tous des produits tensoriels non triviaux, c'est-à-dire sont différents de $S^{p^{k}}$, alors le produit de Yoneda $f \cdot$ e est nul.

Démonstration. On note $\varphi: \operatorname{Id}^{(k)} \rightarrow \mathcal{R}_{G}^{s}$ le représentant de l'extension $f$ défini dans le lemme 3.1 à partir du facteur élémentaire $v$. La construction d'un représentant $\left(\psi: \operatorname{Id}^{(k)} \rightarrow \mathcal{R}_{G}^{s+i}\right)$ de $f \cdot e$ est comme suit. Soit $\mathcal{R}_{\mathrm{Id}^{(k)}}^{\bullet}$ une résolution injective de $\mathrm{Id}^{(k)}$. On peut la choisir telle que $\mathcal{R}_{\mathrm{Id}^{(k)}}^{0}=S^{p^{k}}$. Puisque $\operatorname{Id}^{(k)} \rightarrow \mathcal{R}_{\mathrm{Id}^{(k)}}^{\bullet}$ est acyclique et $G \rightarrow \mathcal{R}_{G}^{\bullet}$ est une résolution injective de $G$, il existe un morphisme de complexes $\mathcal{R}_{\mathrm{Id}^{(k)}}^{\bullet-s} \rightarrow \mathcal{R}_{G}^{\bullet}$ tel que la composition $\mathrm{Id}^{(k)} \rightarrow \mathcal{R}_{\mathrm{Id}^{(k)}}^{0} \rightarrow \mathcal{R}_{G}^{s}$ soit égale à $\varphi$. Soit $\xi: \mathrm{Id}^{(k)} \rightarrow \mathcal{R}_{\mathrm{Id}^{(k)}}^{i}$ un représentant de l'extension $e$. Alors, un représentant de $f \cdot e$ est donné par la composition $\psi: \operatorname{Id}^{(k)} \stackrel{\xi}{\rightarrow} \mathcal{R}_{\mathrm{Id}^{(k)}}^{i} \rightarrow \mathcal{R}_{G}^{s+i}$. 
On construit maintenant explicitement un tel morphisme à l'aide du complexe $\mathcal{R}_{G}^{\bullet}(v)$. Par définition, le morphisme $\varphi$ se factorise au travers de $\mathcal{R}_{G}^{s}(v)=v$. Comme plus haut, il existe alors un morphisme de complexes $\mathcal{R}_{\mathrm{Id}^{(k)}}^{\bullet-s} \rightarrow \mathcal{R}_{G}^{\bullet}(v)$. On obtient un morphisme de complexes $\mathcal{R}_{\mathrm{Id}^{(k)}}^{\bullet-s} \rightarrow \mathcal{R}_{G}^{\bullet}$ en considérant la composition

$$
\mathcal{R}_{\mathrm{Id}^{(k)}}^{\bullet-s} \rightarrow \mathcal{R}_{G}^{\bullet}(v) \rightarrow \mathcal{R}_{G}^{\bullet}
$$

Si $\xi: \operatorname{Id}^{(k)} \rightarrow \mathcal{R}_{\mathrm{Id}^{(k)}}^{i}$ est un représentant de $e$, alors un représentant de $f \cdot e$ est obtenu par la composition $\psi: \mathrm{Id}^{(k)} \stackrel{\xi}{\rightarrow} \mathcal{R}_{\mathrm{Id}^{(k)}}^{i} \rightarrow \mathcal{R}_{G}^{s+i}(v) \rightarrow \mathcal{R}_{G}^{s+i}$.

Si la composition de Yoneda $f \cdot e$ est non nulle, $\psi$ représente une extension non nulle de $\operatorname{Ext}_{\mathcal{P}}^{*}\left(\operatorname{Id}^{(k)}, G\right)$. En appliquant le foncteur $\operatorname{Hom}_{\mathcal{P}}\left(\operatorname{Id}^{(k)},-\right)$ à la composition (3), on obtient une composition de morphismes de complexes

$$
\operatorname{Hom}_{\mathcal{P}}\left(\operatorname{Id}^{(k)}, \mathcal{R}_{\mathrm{Id}^{(k)}}^{\bullet-s}\right) \rightarrow \operatorname{Hom}_{\mathcal{P}}\left(\operatorname{Id}^{(k)}, \mathcal{R}_{G}^{\bullet}(v)\right) \rightarrow \operatorname{Hom}_{\mathcal{P}}\left(\operatorname{Id}^{(k)}, \mathcal{R}_{G}^{\bullet}\right) .
$$

Ces morphismes induisent des morphismes sur la cohomologie en degré $s+i$ :

$$
\begin{aligned}
\mathrm{H}^{s+i}\left(\operatorname{Hom}_{\mathcal{P}}\left(\operatorname{Id}^{(k)}, \mathcal{R}_{\mathrm{Id}^{(n)}}^{\bullet-s}\right)\right) \rightarrow \mathrm{H}^{s+i}\left(\operatorname{Hom}_{\mathcal{P}}\left(\operatorname{Id}^{(k)}, \mathcal{R}_{G}^{\bullet}(v)\right)\right) \\
\rightarrow \mathrm{H}^{s+i}\left(\operatorname{Hom}_{\mathcal{P}}\left(\operatorname{Id}^{(k)}, \mathcal{R}_{G}^{\bullet}\right)\right),
\end{aligned}
$$

ce qui s'écrit aussi

$$
\operatorname{Ext}_{\mathcal{P}}^{i}\left(\operatorname{Id}^{(k)}, \operatorname{Id}^{(k)}\right) \rightarrow \mathrm{H}^{s+i}\left(\operatorname{Hom}_{\mathcal{P}}\left(\operatorname{Id}^{(k)}, \mathcal{R}_{G}^{\bullet}(v)\right)\right) \rightarrow \operatorname{Ext}_{\mathcal{P}}^{s+i}\left(\operatorname{Id}^{(k)}, G\right) .
$$

La composition ci-dessus correspond à la composition de Yoneda (à gauche) par $f$. Puisque $f \cdot e$ est non nul cette composition est non nulle, et donc

$$
\mathrm{H}^{s+i}\left(\operatorname{Hom}_{\mathcal{P}}\left(\operatorname{Id}^{(k)}, \mathcal{R}_{G}^{\bullet}(v)\right)\right) \neq 0 .
$$

Ce groupe est un sous-quotient de $\operatorname{Hom}_{\mathcal{P}}\left(\operatorname{Id}^{(k)}, \mathcal{R}_{G}^{s+i}(v)\right)$. Par le théorème 1.4, $\mathcal{R}_{G}^{s+i}(v)$ contient donc au moins un facteur élémentaire égal à $S^{p^{k}}$.

La proposition 3.2 admet une version réciproque. Pour tout $i \geq 0$, $\operatorname{Ext}_{\mathcal{P}}^{i}\left(\operatorname{Id}^{(k)}, \operatorname{Id}^{(k)}\right)$ est de dimension 0 ou 1 . Si cette dimension est 1 , on note $e(i)$ un élément générateur de $\operatorname{Ext}_{\mathcal{P}}^{i}\left(\operatorname{Id}^{(k)}, \operatorname{Id}^{(k)}\right)$. Dans les autres cas, $e(i)$ désigne l'extension nulle.

Proposition 3.3. Soient $\mathcal{R}_{G}^{\bullet}$ une $S$-résolution explicite réduite d'un foncteur $G \in \mathcal{P}_{p^{k}}$, et $f \in \operatorname{Ext}_{\mathcal{P}}^{s}\left(\operatorname{Id}^{(k)}, G\right)$ une extension représentée par un facteur élémentaire $v_{\mathcal{R}}=S^{p^{k}}$ de $\mathcal{R}_{G}^{s}$. Soit $i$ un entier positif ou nul. Alors si l'extension $f \cdot e$ est nulle, il existe une résolution réduite explicite $\mathcal{T}_{G}^{\bullet}$ de $G$ et un facteur élémentaire $v_{\mathcal{T}}=S^{p^{k}}$ de $\mathcal{T}_{G}^{s}$ représentant l'extension $f$ tels que les sommets de $\Gamma(\{v\}, s+i)$ sont tous différents de $S^{p^{k}}$.

Démonstration. On note $\varphi=\varphi_{v_{\mathcal{R}}}$ le morphisme $\operatorname{Id}^{(k)} \rightarrow \mathcal{R}_{G}^{s}$ égal au morphisme de Frobenius sur $v_{\mathcal{R}}$ et nul sur les autres facteurs. Ce morphisme est un représentant de $f$. 
On commence par construire une $S$-résolution explicite $\mathcal{S}_{G}^{\bullet}$ de $G$ et un morphisme de complexes $g^{\bullet}: \mathcal{S}_{G}^{\bullet} \rightarrow \mathcal{R}_{G}^{\bullet}$ tels que :

(i) pour tout $t<s, \mathcal{R}_{G}^{t}=\mathcal{S}_{G}^{t}$ et $g^{t}$ est l'égalité entre ces termes;

(ii) il existe un facteur élémentaire $v_{\mathcal{S}}=S^{p^{k}}$ de $\mathcal{S}_{G}^{s}$ tel que la restriction de $g^{s}$ à $v_{\mathcal{S}}$ soit un isomorphisme sur $v_{\mathcal{R}}$, et soit nulle sur les autres facteurs de $\mathcal{R}_{G}^{\bullet}$ égaux à $S^{p^{k}}$; en particulier, $v_{\mathcal{S}}$ représente $f$;

(iii) le morphisme $\mathbf{I}_{2}^{s+i, 0}\left(\operatorname{Id}^{(k)}, \mathcal{S}_{G}^{\bullet}\left(v_{\mathcal{S}}\right)\right) \rightarrow \mathbf{I}_{2}^{s+i, 0}\left(\operatorname{Id}^{(k)}, \mathcal{S}_{G}^{\bullet}\right)$ induit par l'inclusion $\mathcal{S}_{G}^{\bullet}\left(v_{\mathcal{S}}\right) \hookrightarrow \mathcal{S}_{G}^{\bullet}$ sur les premières suites spectrales d'hypercohomologie en rang 2 et en degré $(s+i, 0)$ est nul.

La différence essentielle entre la résolution $\mathcal{S}_{G}^{\bullet}$ décrite ci-dessus et la résolution $\mathcal{T}_{G}^{\bullet}$ que l'on veut obtenir au final réside dans le fait que contrairement à $\mathcal{T}_{G}^{\bullet}$, la résolution $\mathcal{S}_{G}^{\bullet}$ n'est pas forcément réduite.

Construction de $\mathcal{S}_{G}^{\bullet}$. On note $K$ le noyau de la différentielle $\mathcal{R}_{G}^{s} \rightarrow \mathcal{R}_{G}^{s+1}$. Comme

$$
d \circ \varphi: \operatorname{Id}^{(k)} \stackrel{\varphi}{\longrightarrow} \mathcal{R}_{G}^{s} \stackrel{d}{\longrightarrow} \mathcal{R}_{G}^{s+1}
$$

est nul, $\varphi$ se factorise par $K$. Soit $\varphi^{\prime}: \operatorname{Id}^{(k)} \rightarrow K$ le morphisme factorisant $\varphi$, et soit $C$ le conoyau de $\varphi^{\prime}$. On obtient une suite exacte courte

$$
0 \rightarrow \mathrm{Id}^{(k)} \stackrel{\varphi^{\prime}}{\longrightarrow} K \rightarrow C \rightarrow 0 .
$$

En effet, le morphisme $\varphi^{\prime}$ est injectif, puisqu'il est non nul, et que $\operatorname{Id}^{(k)}$ est un objet simple de $\mathcal{P}$. Trouver une $S$-résolution $\mathcal{S}_{G}^{\bullet}$ telle que pour tout $t<s$, $\mathcal{S}_{G}^{t}$ soit égal à $\mathcal{R}_{G}^{t}$ revient à trouver une $S$-résolution

$$
K \rightarrow \mathcal{S}_{G}^{s} \rightarrow \mathcal{S}_{G}^{s+1} \rightarrow \ldots
$$

de $K$. On en construit une à partir de la suite exacte (4). Soit $\mathcal{R}_{\mathrm{Id}^{(k)}}$ une $S$-résolution explicite réduite de $\mathrm{Id}^{(k)}$; on la choisit telle que $\mathcal{R}_{\mathrm{Id}^{(k)}}^{0}=S^{p^{k}}$. Soit de même $\mathcal{R}_{C}^{\bullet}$ une $S$-résolution réduite de $C$. Alors $K$ admet une résolution injective $\mathcal{S}_{K}^{\bullet}$ telle que pour tout $t \geq 0$,

$$
\mathcal{S}_{K}^{t}=\mathcal{R}_{\mathrm{Id}^{(k)}}^{t} \oplus \mathcal{R}_{C}^{t}
$$

La résolution injective $\mathcal{S}_{K}^{\bullet}$ de $K$ est une $S$-résolution explicite, la décomposition de $\mathcal{S}_{K}^{t}$ étant donnée par celles de $\mathcal{R}_{\mathrm{Id}^{(k)}}^{t}$ et de $\mathcal{R}_{C}^{t}$. De plus, la différentielle $d_{K}$ de $\mathcal{R}_{K}^{\bullet}$ est égale à la somme de la différentielle $d_{\mathrm{Id}^{(k)}}$ du complexe $\mathcal{R}_{\mathrm{Id}^{(k)}}^{\bullet}$ sur la composante $\mathcal{R}_{\mathrm{Id}^{(k)}}^{\bullet}$, de la différentielle $d_{C}$ du complexe $\mathcal{R}_{C}^{\bullet}$ sur la composante $\mathcal{R}_{C}^{\bullet}$, et d'une partie croisée $\mathcal{R}_{C}^{\bullet} \rightarrow \mathcal{R}_{\mathrm{Id}^{(k)}}^{\bullet+1}$. En revanche, il n'y a pas de composante $\mathcal{R}_{\mathrm{Id}^{(k)}}^{\bullet+1} \rightarrow \mathcal{R}_{C}^{\bullet}$ : 


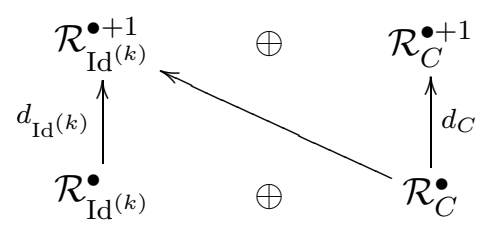

Il en résulte notamment que $\mathcal{R}_{\mathrm{Id}^{(k)}}^{\bullet}$ est un sous-complexe de $\mathcal{S}_{K}^{\bullet}$. La $S$ résolution répondant à la propriété (iii) est alors définie par

$$
\mathcal{S}_{G}^{t}= \begin{cases}\mathcal{R}_{G}^{t} & \text { si } t<s, \\ \mathcal{S}_{K}^{t-s} & \text { si } t \geq s,\end{cases}
$$

avec les différentielles évidentes. L'inclusion de $\mathcal{R}_{\mathrm{Id}^{(k)}}^{\bullet}$ dans $\mathcal{S}_{K}^{\bullet}$ définit une inclusion de $\mathcal{R}_{\mathrm{Id}(k)}^{\bullet-s}$ dans $\mathcal{S}_{G}^{\bullet}$. Le facteur élémentaire $v_{\mathcal{S}}$ est le facteur $S^{p^{k}}$ provenant de $\mathcal{R}_{\mathrm{Id}^{(k)}}^{0}$.

Le morphisme $g^{\bullet}: \mathcal{S}_{G}^{\bullet} \rightarrow \mathcal{R}_{G}^{\bullet}$ est défini par l'identité sur les termes de degré $t<s$; la donnée de ces composantes permet de définir (à homotopie près) le morphisme de complexes $g^{\bullet}$. Le morphisme obtenu vérifie la propriété (ii). En effet, en le composant avec l'inclusion $\mathcal{R}_{\mathrm{Id}^{(k)}}^{\bullet-s} \rightarrow \mathcal{S}_{G}^{\bullet}$, on obtient un diagramme commutatif

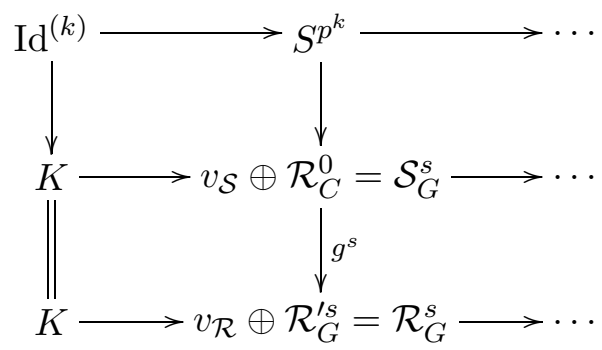

Ici, $\mathcal{R}_{G}^{\prime s}$ est la somme de tous les facteurs élémentaires de $\mathcal{R}_{G}^{s}$ différents de $v_{\mathcal{R}}$. Par définition du morphisme $\operatorname{Id}^{(k)} \rightarrow K$, la composition

$$
\mathrm{Id}^{(k)} \rightarrow K \rightarrow v_{\mathcal{R}} \oplus \mathcal{R}_{G}^{\prime s} \stackrel{\text { proj. }}{\longrightarrow} v_{\mathcal{R}}=S^{p^{k}}
$$

est non nulle. Cela implique notamment que la projection sur $v_{\mathcal{R}}$ de la restriction de $g^{s}: \mathcal{S}_{G}^{s} \rightarrow \mathcal{R}_{G}^{s}$ à $v_{\mathcal{S}}$ est non nulle. Comme c'est un endomorphisme de $S^{p^{k}}$, c'est un isomorphisme. De plus, toujours par définition du morphisme $\mathrm{Id}^{(k)} \rightarrow K$, la composition

$$
\mathrm{Id}^{(k)} \rightarrow K \rightarrow v_{\mathcal{R}} \oplus \mathcal{R}_{G}^{\prime s} \stackrel{\text { proj. }}{\longrightarrow} \mathcal{R}_{G}^{\prime s}
$$

est nulle, d'où la propriété (ii).

Il reste à montrer la propriété (iii). Soit $\Gamma\left(\left\{v_{\mathcal{S}}\right\}\right)$ le sous-graphe de $\Gamma\left(\mathcal{S}_{G}^{\bullet}\right)$ engendré par le sommet $v_{\mathcal{S}}$. Puisque $\mathcal{R}_{\mathrm{Id}^{(k)}}^{\bullet-s}$ est un sous-complexe de $\mathcal{S}_{G}^{\bullet}$, le graphe $\Gamma\left(\left\{v_{\mathcal{S}}\right\}\right)$ est le sous-graphe de $\Gamma\left(\mathcal{R}_{\mathrm{Id}^{(k)}}^{\bullet-s}\right)$ engendré par le sommet $v_{\mathcal{S}}$ en degré $s$. Comme $\mathcal{R}_{\mathrm{Id}^{(k)}}^{\bullet}$ est une résolution réduite de $\mathrm{Id}^{(k)}$, elle possède, 
d'après le théorème 1.3 , exactement un facteur élémentaire égal à $S^{p^{k}}$ en degré pair strictement inférieur à $2 p^{k}$, et aucun dans les autres degrés. Ainsi, $\Gamma\left(\left\{v_{\mathcal{S}}\right\}\right)$ a au plus un sommet égal à $S^{p^{k}}$ en degré $s+2 t, 0 \leq t<p^{k}$, et n'en a aucun dans les autres degrés.

- Si $e(i)$ est nulle (soit $i$ est impair, soit $i \geq 2 p^{k}$ ), l'argument ci-dessus montre qu'il n'y a pas de facteur élémentaire égal à $S^{p^{k}}$ dans l'ensemble de sommets $\Gamma\left(\left\{v_{\mathcal{S}}\right\}, s+i\right)$, et par conséquent

$$
\mathbf{I}_{1}^{i, 0}\left(\operatorname{Id}^{(k)}, \mathcal{S}_{G}^{\bullet}\left(\left\{v_{\mathcal{S}}\right\}\right)\right)=0,
$$

ce qui implique immédiatement la propriété (iii).

- Supposons que $e(i)$ est non nulle, et que $f \cdot e(i)=0$. L'extension $e(i)$ est représentée, à un scalaire près, par le morphisme $\xi: \mathrm{Id}^{(k)} \rightarrow \mathcal{R}_{\mathrm{Id}^{(k)}}^{i}$ égal au morphisme de Frobenius sur le seul facteur élémentaire égal à $S^{p^{k}}$, et égal à 0 sinon. Pour que $\mathcal{S}_{G}^{\bullet}$ vérifie la propriété (iii), il faut montrer que le morphisme

$$
\mathbf{I}_{2}^{s+i, 0}\left(\operatorname{Id}^{(k)}, \mathcal{S}_{G}^{\bullet}\left(v_{\mathcal{S}}\right)\right) \rightarrow \mathbf{I}_{2}^{s+i, 0}\left(\operatorname{Id}^{(k)}, \mathcal{S}_{G}^{\bullet}\right)
$$

est nul. Si $\mathbf{I}_{1}^{s+i, 0}\left(\operatorname{Id}^{(k)}, \mathcal{S}_{G}^{\bullet}\left(v_{\mathcal{S}}\right)\right)$ est nul, la propriété (iii) est évidente. Sinon, $\Gamma\left(\left\{v_{\mathcal{S}}\right\}, s+i\right)$ possède au moins (puis exactement) un sommet égal à $S^{p^{k}}$, qui correspond au facteur élémentaire $S^{p^{k}}$ de $\mathcal{R}_{\mathrm{Id}^{(k)}}^{i}$ représentant $e(i)$. Ainsi, le morphisme $\xi$ se factorise à travers $\mathcal{S}_{G}^{s+i}\left(v_{\mathcal{S}}\right)$. Alors, dire que le morphisme (6) est nul revient à dire que le morphisme de $\operatorname{Hom}_{\mathcal{P}}\left(\operatorname{Id}^{(k)}, \mathcal{S}_{G}^{s+i}\right)$ défini par la composition

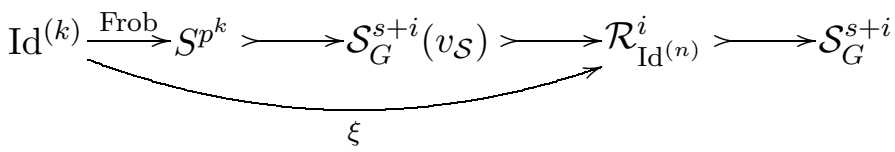

est un représentant de l'extension nulle de $\operatorname{Ext}_{\mathcal{P}}^{s+i}\left(\operatorname{Id}^{(k)}, G\right)$, ce qui est le cas, puisque ce morphisme représente l'extension $f \cdot[\xi]=f \cdot e(i)$.

Construction de $\mathcal{T}_{G}^{\bullet}$. On peut maintenant construire une $S$-résolution réduite $\mathcal{T}_{G}^{\bullet}$ de $G$ qui répond aux exigences de la proposition 3.3. En considérant la suite exacte longue associée à la suite exacte courte

$$
0 \rightarrow \mathrm{Id}^{(k)} \rightarrow K \rightarrow C \rightarrow 0
$$

on obtient, puisque $\operatorname{Ext}_{\mathcal{P}}^{1}\left(\operatorname{Id}^{(k)}, \operatorname{Id}^{(k)}\right)$ est nul, une suite exacte courte

$$
0 \rightarrow \operatorname{Ext}_{\mathcal{P}}^{0}\left(\operatorname{Id}^{(k)}, \operatorname{Id}^{(k)}\right) \rightarrow \operatorname{Ext}_{\mathcal{P}}^{0}\left(\operatorname{Id}^{(k)}, K\right) \rightarrow \operatorname{Ext}_{\mathcal{P}}^{0}\left(\operatorname{Id}^{(k)}, C\right) \rightarrow 0 .
$$

Par conséquent,

$$
\operatorname{dim} \operatorname{Ext}_{\mathcal{P}}^{0}\left(\operatorname{Id}^{(k)}, K\right)=\operatorname{dim} \operatorname{Ext}_{\mathcal{P}}^{0}\left(\operatorname{Id}^{(k)}, \operatorname{Id}^{(k)}\right)+\operatorname{dim} \operatorname{Ext}_{\mathcal{P}}^{0}\left(\operatorname{Id}^{(k)}, C\right) .
$$

Ainsi, puisque $\mathcal{R}_{C}^{\bullet}$ est une résolution réduite de $C, K \rightarrow \mathcal{S}_{K}^{0}=S^{p^{k}} \oplus \mathcal{R}_{C}^{0}$ 
est le début d'une $S$-résolution réduite explicite de $K$. On la complète en une $S$-résolution réduite explicite $\mathcal{T}_{K}^{\bullet}$ de $K$, où $\mathcal{T}_{K}^{0}=\mathcal{S}_{K}^{0}$. On définit alors $\mathcal{T}_{G}^{\bullet}$ par

$$
\mathcal{T}_{G}^{t}= \begin{cases}\mathcal{R}_{G}^{t} & \text { si } t<s, \\ \mathcal{T}_{K}^{t-s} & \text { si } t \geq s .\end{cases}
$$

On obtient ainsi une $S$-résolution réduite explicite $\mathcal{T}_{G}^{\bullet}$ de $G$ telle que $\mathcal{T}_{G}^{t}=\mathcal{S}_{G}^{t}$ pour tout $t \leq s$. En particulier, le facteur élémentaire $v_{\mathcal{S}}$ de $\mathcal{S}_{G}^{s}$ est aussi un facteur élémentaire de $\mathcal{T}_{G}^{s}$, que l'on notera $v_{\mathcal{T}}$. Il existe un morphisme de complexes $h^{\bullet}: \mathcal{T}_{G}^{\bullet} \rightarrow \mathcal{S}_{G}^{\bullet}$ tel que $h^{t}: \mathcal{T}_{G}^{t} \rightarrow \mathcal{S}_{G}^{t}$ soit l'identité pour tout $t \leq s$. Notamment, la restriction du morphisme $h^{\bullet}$ à $v_{\mathcal{T}}$ est un isomorphisme sur le facteur $v_{\mathcal{S}}$, et nul sur les autres facteurs élémentaires. Par conséquent, $h^{\bullet}$ induit un morphisme de complexes $\widetilde{h}^{\bullet}: \mathcal{T}_{G}^{\bullet}\left(v_{\mathcal{T}}\right) \rightarrow \mathcal{S}_{G}^{\bullet}\left(v_{\mathcal{S}}\right)$ tel que le carré suivant soit commutatif :

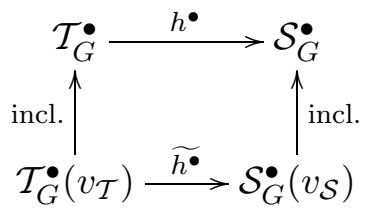

Ce carré commutatif induit un carré commutatif de morphismes de suites spectrales sur les premières suites spectrales d'hypercohomologie $\mathbf{I}\left(\operatorname{Id}^{(k)},-\right)$ :

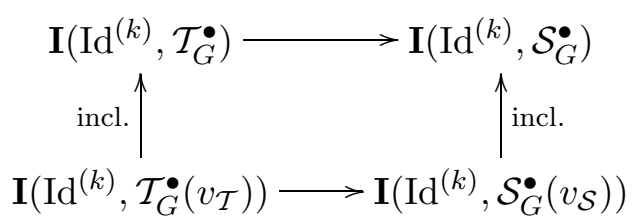

Toutes ces suites spectrales sont nulles ailleurs que sur leur ligne 0. De plus :

- comme $\mathcal{T}_{G}^{\bullet}$ et $\mathcal{S}_{G}^{\bullet}$ sont deux résolutions injectives du même objet $G$, $\mathbf{I}_{2}^{*, 0}\left(\operatorname{Id}^{(k)}, \mathcal{T}_{G}^{\bullet}\right)$ et $\mathbf{I}_{2}^{*, 0}\left(\operatorname{Id}^{(k)}, \mathcal{S}_{G}^{\bullet}\right)$ sont égaux à $\operatorname{Ext}_{\mathcal{P}}^{*}\left(\operatorname{Id}^{(k)}, G\right)$, et le morphisme du haut est un isomorphisme;

- en rang 1, le morphisme de gauche $\mathbf{I}_{1}^{*, 0}\left(\operatorname{Id}^{(k)}, \mathcal{T}_{G}^{\bullet}\left(v_{\mathcal{T}}\right)\right) \rightarrow \mathbf{I}_{1}^{*, 0}\left(\operatorname{Id}^{(k)}, \mathcal{T}_{G}^{\bullet}\right)$ est le morphisme $\operatorname{Ext}_{\mathcal{P}}^{*}\left(\operatorname{Id}^{(k)}, \mathcal{T}_{G}^{t}\left(v_{\mathcal{T}}\right)\right) \rightarrow \operatorname{Ext}_{\mathcal{P}}^{*}\left(\operatorname{Id}^{(k)}, \mathcal{T}_{G}^{t}\right)$ induit par l'inclusion directe $\mathcal{T}_{G}^{t}\left(v_{\mathcal{T}}\right) \rightarrow \mathcal{T}_{G}^{t}$, il est donc injectif;

- ce morphisme reste une injection en rang 2, car la suite spectrale $\mathbf{I}\left(\operatorname{Id}^{(k)}, \mathcal{T}_{G}^{\bullet}\right)$ dégénère au rang 1 , la $S$-résolution $\mathcal{T}_{G}^{\bullet}$ étant réduite;

- par la propriété (iii) pour $\mathcal{S}_{G}^{\bullet}$, le morphisme de droite est nul au rang 0 en degré $(s+i, 0)$. 
On en déduit un diagramme commutatif

$$
\begin{aligned}
\mathbf{I}_{2}^{s+i, 0}\left(\operatorname{Id}^{(k)}, \mathcal{T}_{G}^{\bullet}\right) & \simeq \mathbf{I}_{2}^{s+i, 0}\left(\operatorname{Id}^{(k)}, \mathcal{S}_{G}^{\bullet}\right) \\
\mathbf{I}_{2}^{s+i, 0}\left(\operatorname{Id}^{(k)}, \mathcal{T}_{G}^{\bullet}\left(v_{\mathcal{T}}\right)\right) & \longrightarrow \mathbf{I}_{2}^{s+i, 0}\left(\operatorname{Id}^{(k)}, \mathcal{S}_{G}^{\bullet}\left(v_{\mathcal{S}}\right)\right)
\end{aligned}
$$

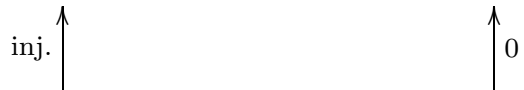

Par conséquent, le morphisme $\mathbf{I}_{2}^{s+i, 0}\left(\operatorname{Id}^{(k)}, \mathcal{T}_{G}^{\bullet}\left(v_{\mathcal{T}}\right)\right) \rightarrow \mathbf{I}_{2}^{s+i, 0}\left(\operatorname{Id}^{(k)}, \mathcal{S}_{G}^{\bullet}\right)$ obtenu en composant des deux manières possibles les flèches du carré (7) est à la fois nul et injectif. Ainsi,

$$
\mathbf{I}_{2}^{s+i, 0}\left(\operatorname{Id}^{(k)}, \mathcal{T}_{G}^{\bullet}\left(v_{\mathcal{T}}\right)\right)=0, \quad \text { puis } \quad \operatorname{Ext}_{\mathcal{P}}^{0}\left(\operatorname{Id}^{(k)}, \mathcal{T}_{G}^{s+i}\left(v_{\mathcal{T}}\right)\right)=0,
$$

puisque les différentielles de rang 1 sont nulles, du fait que $\mathcal{T}_{G}^{\bullet}$ est réduite. Par le théorème de Pirashvili (théorème 1.4), on en déduit que tous les facteurs élémentaires de $\mathcal{T}_{G}^{s+i}\left(v_{\mathcal{T}}\right)$ sont différents de $S^{p^{k}}$.

Remarque 3.4. La construction de $\mathcal{T}_{G}^{\bullet}$ ne dépend pas du degré $i$ de l'extension $e(i)$ considérée dans l'énoncé. On n'utilise l'entier $i$ qu'au moment de vérifier qu'on a les propriétés requises pour la résolution $\mathcal{T}_{G}^{\bullet}$. Ainsi, on peut faire le même raisonnement simultanément sur plusieurs indices $i_{1}, \ldots, i_{k}$ :

Proposition 3.5. Soit $f$ un objet de $\operatorname{Ext}_{\mathcal{P}}^{s}\left(\operatorname{Id}^{(k)}, G\right)$. Soient $i_{1}<\ldots<i_{l}$ des entiers tels que pour tout $j$ vérifiant $1 \leq j \leq l$, le produit $f \cdot e\left(i_{j}\right)$ est nul. Alors il existe une $S$-résolution réduite explicite $\mathcal{T}_{G}^{\bullet}$, et un facteur élémentaire $v_{\mathcal{T}}=S^{p^{k}}$ de $\mathcal{T}_{G}^{s}$ représentant $f$, tels que tous les sommets de $\Gamma\left(\left\{v_{\mathcal{T}}\right\}\right)$ en degrés $s+i_{1}, \ldots, s+i_{l}$ sont différents de $S^{p^{k}}$.

4. Graphes et différentielles. Soient $F$ et $G$ deux objets de $\mathcal{P}$, homogènes de degré respectivement $p^{h}$ et $p^{k}$. Soit $\mathcal{R}_{G}^{\bullet}$ une $S$-résolution réduite de $G$, et $v_{\mathcal{R}}=S^{p^{k}}$ un facteur élémentaire de $\mathcal{R}_{G}^{s}$ représentant une extension $f \in \operatorname{Ext}_{\mathcal{P}}^{s}\left(\operatorname{Id}^{(k)}, G\right)$. Le complexe $\mathcal{R}_{G}^{\bullet} \circ F$ obtenu en précomposant $\mathcal{R}_{G}^{\bullet}$ par $F$ est une résolution (non injective) de $G \circ F$, puisque la précomposition est exacte. Ainsi, sa première suite spectrale $\mathbf{I}\left(\operatorname{Id}^{(h+k)}, \mathcal{R}_{G}^{\bullet} \circ F\right)$ converge vers $\operatorname{Ext}_{\mathcal{P}}^{*}\left(\operatorname{Id}^{(h+k)}, G \circ F\right)$. De plus, puisque la résolution $\mathcal{R}_{G}^{\bullet}$ est réduite, elle est décrite en rang 1 par l'isomorphisme bigradué

(8) $\mathbf{I}_{1}^{*_{1}, *_{2}}\left(\operatorname{Id}^{(h+k)}, \mathcal{R}_{G}^{\bullet} \circ F\right)=\operatorname{Ext}_{\mathcal{P}}^{*_{1}}\left(\operatorname{Id}^{(k)}, G\right) \otimes \operatorname{Ext}_{\mathcal{P}}^{*_{2}}\left(\operatorname{Id}^{(h+k)}, S^{p^{k}} \circ F\right)$.

Autrement dit, chaque colonne d'abscisse $t$ est constituée d'autant de copies de $\operatorname{Ext}_{\mathcal{P}}^{*}\left(\operatorname{Id}^{(h+k)}, S^{p^{k}} \circ F\right)$ qu'il y a de facteurs élémentaires $S^{p^{k}}$ dans $\mathcal{R}_{G}^{t}$. En particulier, au facteur $v_{\mathcal{R}}$ correspond une copie

$$
\operatorname{Ext}_{\mathcal{P}}^{*}\left(\operatorname{Id}^{(h+k)}, v_{\mathcal{R}} \circ F\right)=\operatorname{Ext}_{\mathcal{P}}^{*}\left(\operatorname{Id}^{(h+k)}, S^{p^{k}} \circ F\right) .
$$

Dans la notation de l'équation (8), les éléments de cette copie correspondent aux éléments $f \otimes f^{\prime}$, pour $f^{\prime} \in \operatorname{Ext}_{\mathcal{P}}^{*}\left(\operatorname{Id}^{(h+k)}, S^{p^{k}} \circ F\right)$. 
Proposition 4.1. Soit $t$ un entier strictement positif. Dans le contexte ci-dessus, si pour tout $i$ tel que $0<i \leq t$, le produit $f \cdot e(i)$ est nul, alors les différentielles de rang inférieur ou égal à $t$ de $\mathbf{I}_{1}\left(\mathrm{Id}^{(h+k)}, \mathcal{R}_{G}^{\bullet} \circ F\right)$ sont nulles sur les éléments $f \otimes f^{\prime}$, pour tout $f^{\prime} \in \operatorname{Ext}_{\mathcal{P}}^{*}\left(\operatorname{Id}^{(h+k)}, S^{p^{k}} \circ F\right)$, et leurs images en rangs supérieurs.

Démonstration. D'après la proposition 3.5 , il existe une $S$-résolution réduite explicite $\mathcal{T}_{G}^{\bullet}$ de $G$ telle que

(i) $\mathcal{T}_{G}^{u}=\mathcal{R}_{G}^{u}$ si $u<s$;

(ii) il existe un facteur élémentaire $v_{\mathcal{T}}$ de $\mathcal{T}_{G}^{s}$ et un morphisme de complexes $g^{\bullet}: \mathcal{T}_{G}^{\bullet} \rightarrow \mathcal{R}_{G}^{\bullet}$ tels que $g^{u}$ est l'identité si $u<s$, et la restriction de $g^{s}$ à $v_{\mathcal{T}}$ est un isomorphisme sur le facteur $v_{\mathcal{R}}$ de $\mathcal{R}_{G}^{\bullet}$, et est nulle sur les autres facteurs élémentaires égaux à $S^{p^{k}}$;

(iii) le sous-graphe $\Gamma\left(v_{\mathcal{T}}\right)$ de $\Gamma\left(\mathcal{T}_{G}^{\bullet}\right)$ ne contient pas de sommet égal à $S^{p^{k}}$, en tout degré $i$ tel que $1 \leq i \leq t$.

Le morphisme $g^{\bullet} \circ F: \mathcal{T}_{G}^{\bullet} \circ F \rightarrow \mathcal{R}_{G}^{\bullet} \circ F$ induit un isomorphisme de suites spectrales $\mathbf{I}\left(\mathrm{Id}^{(h+k)}, \mathcal{T}_{G}^{\bullet} \circ F\right) \stackrel{\simeq}{\rightarrow} \mathbf{I}\left(\mathrm{Id}^{(h+k)}, \mathcal{R}_{G}^{\bullet} \circ F\right) \grave{a}$ partir du rang 2 . Puisque les résolutions $\mathcal{R}_{G}^{\bullet}$ et $\mathcal{T}_{G}^{\bullet}$ sont réduites, c'est en fait un isomorphisme à partir du rang 1 . De plus, grâce à la propriété (ii) pour $\mathcal{T}_{G}^{\bullet}$, la restriction du morphisme induit par $g^{\bullet} \circ F$ en rang 1 au facteur direct $\operatorname{Ext}_{\mathcal{P}}^{*}\left(\operatorname{Id}^{(k)}, v_{\mathcal{T}} \circ F\right)$ de $\mathbf{I}_{1}\left(\operatorname{Id}^{(k)}, \mathcal{T}_{G}^{\bullet} \circ F\right)$ est un isomorphisme sur le facteur $\operatorname{Ext}_{\mathcal{P}}^{*}\left(\operatorname{Id}^{(k)}, v_{\mathcal{R}} \circ F\right)$ de $\mathbf{I}_{1}\left(\operatorname{Id}^{(k)}, \mathcal{R}_{G}^{\bullet} \circ F\right)$, et nul sur les autres facteurs. Autrement dit, le morphisme $g^{\bullet} \circ F$ induit l'identité sur les termes $f \otimes f^{\prime}, f^{\prime} \in \operatorname{Ext}_{\mathcal{P}}^{*}\left(\operatorname{Id}^{(h+k)}, S^{p^{k}} \circ F\right)$.

Il suffit donc de montrer que les différentielles de rang inférieur ou égal à $t$ de la suite spectrale $\mathbf{I}\left(\operatorname{Id}^{(h+k)}, \mathcal{T}_{G}^{\bullet} \circ F\right)$ sont nulles sur les termes $f \otimes f^{\prime}$, pour $f^{\prime} \in \operatorname{Ext}_{\mathcal{P}}^{*}\left(\operatorname{Id}^{(h+k)}, S^{p^{k}} \circ F\right)$. L'inclusion $\mathcal{T}_{G}^{\bullet}\left(v_{\mathcal{T}}\right) \rightarrow \mathcal{T}_{G}^{\bullet}$ induit un morphisme de suites spectrales

$$
\mathbf{I}\left(\operatorname{Id}^{(h+k)}, \mathcal{T}_{G}^{\bullet}\left(v_{\mathcal{T}}\right) \circ F\right) \rightarrow \mathbf{I}\left(\operatorname{Id}^{(h+k)}, \mathcal{T}_{G}^{\bullet} \circ F\right) .
$$

Sur les termes $\mathbf{I}_{1}$, ce morphisme est une inclusion : sur la colonne d'abscisse $t$, c'est le morphisme $\operatorname{Ext}_{\mathcal{P}}^{*}\left(\operatorname{Id}^{(h+k)}, \mathcal{T}_{G}^{t}\left(v_{\mathcal{T}}\right) \circ F\right) \rightarrow \operatorname{Ext}_{\mathcal{P}}^{*}\left(\operatorname{Id}^{(h+k)}, \mathcal{T}_{G}^{t} \circ F\right)$ induit par l'inclusion directe $\mathcal{T}_{G}^{t}\left(v_{\mathcal{T}}\right) \rightarrow \mathcal{T}_{G}^{t}$. Par exemple, sur la colonne d'abscisse $s$, il consiste en l'inclusion directe

$$
\begin{aligned}
& \operatorname{Ext}_{\mathcal{P}}^{*}\left(\operatorname{Id}^{(h+k)}, v_{\mathcal{T}} \circ F\right)=\operatorname{Ext}_{\mathcal{P}}^{*}\left(\operatorname{Id}^{(h+k)}, S^{p^{k}} \circ F\right) \rightarrow \operatorname{Ext}_{\mathcal{P}}^{*}\left(\operatorname{Id}^{(h+k)}, \mathcal{T}_{G}^{s} \circ F\right), \\
& f^{\prime} \mapsto f \otimes f^{\prime} .
\end{aligned}
$$

D'après la proposition 3.5 et le théorème de Pirashvili, les colonnes d'abscisse $j$ telle que $s<j \leq s+t$ de la suite spectrale $\mathbf{I}\left(\operatorname{Id}^{(h+k)}, \mathcal{T}_{G}^{\bullet}\left(v_{\mathcal{T}}\right) \circ F\right)$ sont nulles. Ainsi, les différentielles de rang $r \leq t$ issues de la colonne d'abscisse $s$ sont nulles; les différentielles dont le but est dans cette colonne 
sont aussi nulles. Par conséquent,

$$
\begin{aligned}
\mathbf{I}_{1}^{s, *}\left(\operatorname{Id}^{(h+k)}, \mathcal{T}_{G}^{\bullet}\left(v_{\mathcal{T}}\right) \circ F\right) & =\mathbf{I}_{2}^{s, *}\left(\operatorname{Id}^{(h+k)}, \mathcal{T}_{G}^{\bullet}\left(v_{\mathcal{T}}\right) \circ F\right)=\ldots \\
& =\mathbf{I}_{t+1}^{s, *}\left(\operatorname{Id}^{(h+k)}, \mathcal{T}_{G}^{\bullet}\left(v_{\mathcal{T}}\right) \circ F\right) .
\end{aligned}
$$

En particulier, le morphisme (9) est en tout rang $r \leq t$ une surjection de la colonne $s$ vers l'espace engendré par les images en rang supérieur des éléments $f \otimes f^{\prime}, f^{\prime} \in \operatorname{Ext}_{\mathcal{P}}^{*}\left(\operatorname{Id}^{(h+k)}, S^{p^{k}} \circ F\right)$. Ainsi, pour montrer que les différentielles de rang $1, \ldots, t$ sont nulles sur $f \otimes f^{\prime}$, il suffit de montrer que pour tout $r \leq t$, la composition

$$
\begin{aligned}
\mathbf{I}_{r}^{s, *}\left(\operatorname{Id}^{(h+k)}, \mathcal{T}_{G}^{\bullet}\left(v_{\mathcal{T}}\right) \circ F\right) \rightarrow \mathbf{I}_{r}^{s, *}\left(\operatorname{Id}^{(h+k)},\right. & \left.\mathcal{T}_{G}^{\bullet} \circ F\right) \\
& \stackrel{d_{r}}{\longrightarrow} \mathbf{I}_{r}^{s+r, *-r+1}\left(\operatorname{Id}^{(h+k)}, \mathcal{T}_{G}^{\bullet} \circ F\right)
\end{aligned}
$$

est nulle. Cela provient du carré commutatif suivant :

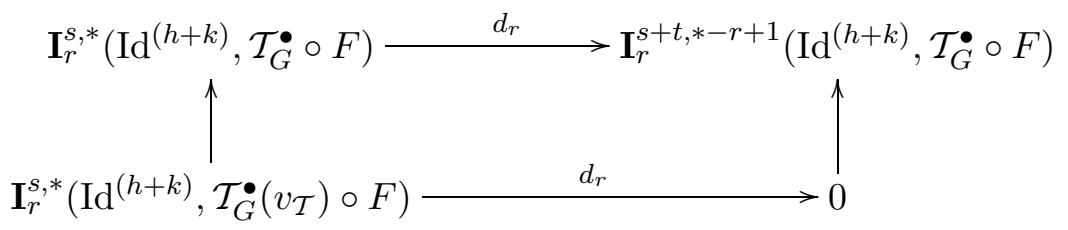

La proposition 4.1 admet une version jumelle en postcomposant par $F$ au lieu de précomposer. On gère le défaut d'exactitude de la postcomposition grâce au théorème 1.6 : la suite spectrale $\mathbf{I}_{1}\left(\mathrm{Id}^{(h+k)}, F \circ \mathcal{R}_{G}^{\bullet}\right)$ est comme précédemment donnée au rang 1 par

$$
\mathbf{I}_{1}^{*_{1}, *_{2}}\left(\operatorname{Id}^{(h+k)}, F \circ \mathcal{R}_{G}^{\bullet}\right)=\operatorname{Ext}_{\mathcal{P}}^{*_{1}}\left(\operatorname{Id}^{(k)}, G\right) \otimes \operatorname{Ext}_{\mathcal{P}}^{*_{2}}\left(\operatorname{Id}^{(h+k)}, F \circ S^{p^{k}}\right) .
$$

On obtient, par la même démonstration, en faisant appel au théorème 1.5 :

Proposition 4.2. Soitt un entier strictement positif. Si pour tout $i \leq t$, le produit $f \cdot e(i)$ est nul, alors les différentielles de rang inférieur ou égal à $t$ de la suite spectrale $\mathbf{I}\left(\mathrm{Id}^{(h+k)}, F \circ \mathcal{R}_{G}^{\bullet}\right)$ sont nulles sur les éléments $f \otimes f^{\prime}$, $f^{\prime} \in \operatorname{Ext}_{\mathcal{P}}^{*}\left(\operatorname{Id}^{(h+k)}, F \circ S^{p^{k}}\right)$.

5. Une formule pour $\operatorname{Ext}_{\mathcal{P}}^{*}(\mathrm{Id}, G \circ F)$. Nous avons maintenant tous les outils nécessaires pour démontrer le théorème $\mathrm{A}$. Nous montrons dans un premier temps que la formule du théorème $\mathrm{A}$ est un isomorphisme d'espaces vectoriels gradués :

ThÉORÈme 5.1. Soient $F$ et $G$ deux objets de $\mathcal{P}$, homogènes de degrés respectifs $p^{h}$ et $p^{k}$. On suppose que $\operatorname{Ext}_{\mathcal{P}}^{*}\left(\operatorname{Id}^{(h)}, F\right)$ et $\operatorname{Ext}_{\mathcal{P}}^{*}\left(\operatorname{Id}^{(k)}, G\right)$ ont une structure de module de Yoneda triviale. Alors, en tant qu'espace vectoriel gradué,

$$
\begin{aligned}
& \operatorname{Ext}_{\mathcal{P}}^{*}\left(\operatorname{Id}^{(h+k)}, G \circ F\right) \\
& \quad \cong \operatorname{Ext}_{\mathcal{P}}^{*}\left(\operatorname{Id}^{(h)}, F\right) \otimes \operatorname{Ext}_{\mathcal{P}}^{*}\left(\operatorname{Id}^{(k)}, G\right) \otimes \operatorname{Ext}_{\mathcal{P}}^{*}\left(\operatorname{Id}^{(h+k)}, S^{p^{k}} \circ S^{p^{h}}\right) .
\end{aligned}
$$


Démonstration. Soit $\mathcal{R}_{G}^{\bullet}$ une $S$-résolution réduite explicite de $G$, et $\mathcal{R}_{G}^{\bullet} \circ F$ le complexe obtenu en précomposant la résolution $\mathcal{R}_{G}^{\bullet}$ par $F$. Puisque la précomposition est exacte, la seule cohomologie de ce complexe est $G \circ F$ en degré 0 . Par conséquent, sa seconde suite spectrale d'hypercohomologie est donnée par

$$
\mathbf{I I}_{2}^{*, t}\left(\operatorname{Id}^{(h+k)}, \mathcal{R}_{G}^{\bullet} \circ F\right)= \begin{cases}\operatorname{Ext}_{\mathcal{P}}^{*}\left(\operatorname{Id}^{(h+k)}, G \circ F\right) & \text { si } t=0, \\ 0 & \text { sinon. }\end{cases}
$$

En particulier, elle dégénère au rang 2, et la cohomologie totale est égale au module $\operatorname{Ext}_{\mathcal{P}}^{*}\left(\operatorname{Id}^{(h+k)}, G \circ F\right)$. C'est aussi l'aboutissement de la première suite spectrale d'hypercohomologie $\mathbf{I}\left(\operatorname{Id}^{(h+k)}, \mathcal{R}_{G}^{\bullet} \circ F\right)$. Puisque, d'après le théorème de Pirashvili (théorème 1.4), $\operatorname{Ext}_{\mathcal{P}}^{*}\left(\operatorname{Id}^{(h+k)}, w \circ F\right)=0$ pour tout facteur élémentaire $w$ de $\mathcal{R}_{G}^{\bullet}$ différent de $S^{p^{k}}$, celle-ci est donnée en rang 1 par

$$
\mathbf{I}_{1}^{s, *}\left(\operatorname{Id}^{(h+k)}, \mathcal{R}_{G}^{\bullet} \circ F\right)=\left[\operatorname{Ext}_{\mathcal{P}}^{*}\left(\operatorname{Id}^{(h+k)}, S^{p^{k}} \circ F\right)\right]^{\oplus d_{s}},
$$

où $d_{s}$ est le nombre de facteurs élémentaires égaux à $S^{p^{k}}$ de $\mathcal{R}_{G}^{s}$. Comme la $S$-résolution $\mathcal{R}_{G}^{\bullet}$ est réduite, $d_{s}$ est la dimension de $\operatorname{Ext}_{\mathcal{P}}^{s}\left(\operatorname{Id}^{(k)}, G\right)$. Ainsi, en tant qu'espace vectoriel bigradué,

$$
\mathbf{I}_{1}^{*_{1}, *_{2}}\left(\operatorname{Id}^{(h+k)}, \mathcal{R}_{G}^{\bullet} \circ F\right) \cong \operatorname{Ext}_{\mathcal{P}}^{*_{1}}\left(\operatorname{Id}^{(k)}, G\right) \otimes \operatorname{Ext}_{\mathcal{P}}^{*_{2}}\left(\operatorname{Id}^{(h+k)}, S^{p^{k}} \circ F\right) .
$$

Mais puisque $\operatorname{Ext}_{\mathcal{P}}^{*}\left(\operatorname{Id}^{(k)}, G\right)$ est un module trivial, toutes les différentielles de $\mathbf{I}\left(\operatorname{Id}^{(h+k)}, \mathcal{R}_{G}^{\bullet} \circ F\right)$ sont nulles d'après la proposition 4.1. Ainsi, la suite spectrale $\mathbf{I}\left(\operatorname{Id}^{(h+k)}, \mathcal{R}_{G}^{\bullet} F\right)$ dégénère au rang 1 , et l'isomorphisme (10) donne à l'aboutissement un isomorphisme d'espaces vectoriels gradués

$$
\operatorname{Ext}_{\mathcal{P}}^{*}\left(\operatorname{Id}^{(h+k)}, G \circ F\right) \cong \operatorname{Ext}_{\mathcal{P}}^{*}\left(\operatorname{Id}^{(k)}, G\right) \otimes \operatorname{Ext}_{\mathcal{P}}^{*}\left(\operatorname{Id}^{(h+k)}, S^{p^{k}} \circ F\right) .
$$

On raisonne de la même façon pour exprimer $\operatorname{Ext}_{\mathcal{P}}^{*}\left(\operatorname{Id}^{(h+k)}, S^{p^{k}} \circ F\right)$ en fonction de $\operatorname{Ext}_{\mathcal{P}}^{*}\left(\operatorname{Id}^{(h)}, F\right)$ et $\operatorname{Ext}_{\mathcal{P}}^{*}\left(\operatorname{Id}^{(h+k)}, S^{p^{k}} \circ S^{p^{h}}\right)$, à la différence près que maintenant on postcompose une résolution $\mathcal{R}_{F}^{\bullet}$ de $F$ par $S^{p^{k}}$. En utilisant le théorème 1.6 pour déterminer la seconde suite spectrale d'hypercohomologie, et le théorème 1.5 pour déterminer la première suite spectrale, on obtient de la même façon que ci-dessus

$$
\operatorname{Ext}_{\mathcal{P}}^{*}\left(\operatorname{Id}^{(h+k)}, S^{p^{k}} \circ F\right) \cong \operatorname{Ext}_{\mathcal{P}}^{*}\left(\operatorname{Id}^{(k)}, F\right) \otimes \operatorname{Ext}_{\mathcal{P}}^{*}\left(\operatorname{Id}^{(h+k)}, S^{p^{k}} \circ S^{p^{h}}\right) .
$$

Les isomorphismes (11) et (12) donnent la formule du théorème 5.1.

THÉORÈmE 5.2. La formule du théorème 5.1 est un isomorphisme de modules de Yoneda sur l'algèbre $\operatorname{Ext}_{\mathcal{P}}^{*}\left(\mathrm{Id}^{(h+k)}, \mathrm{Id}^{(h+k)}\right)$, la structure de module du produit tensoriel provenant de la structure de module du troisième terme.

Démonstration. On montre dans un premier temps que l'isomorphisme (11) est un isomorphisme de modules, la structure de module du 
produit tensoriel provenant de la structure de module du second facteur. Le problème vient de ce que le produit tensoriel de $f \in \operatorname{Ext}_{\mathcal{P}}^{s}\left(\operatorname{Id}^{(k)}, G\right)$ et $g \in \operatorname{Ext}_{\mathcal{P}}^{t}\left(\operatorname{Id}^{(h+k)}, S^{p^{k}} \circ F\right)$ n'est a priori pas bien défini dans l'aboutissement $\operatorname{Ext}_{\mathcal{P}}^{s+t}\left(\operatorname{Id}^{(h+k)}, G \circ F\right)$ de la suite spectrale $\mathbf{I}\left(\operatorname{Id}^{(h+k)}, \mathcal{R}_{G}^{\bullet} \circ F\right)$, car on n'obtient qu'un gradué de la cohomologie totale. On va construire explicitement un élément $f \otimes g$ de $\operatorname{Ext}_{\mathcal{P}}^{s+t}\left(\operatorname{Id}^{(h+k)}, G \circ F\right)$ tel que

(i) l'application $(f, g) \mapsto f \otimes g$ soit bilinéaire;

(ii) l'application

$$
\begin{aligned}
\operatorname{Ext}_{\mathcal{P}}^{*}\left(\operatorname{Id}^{(k)}, G\right) \otimes \operatorname{Ext}_{\mathcal{P}}^{*}\left(\operatorname{Id}^{(h+k)}, S^{p^{k}} \circ F\right) & \rightarrow \operatorname{Ext}_{\mathcal{P}}^{*}\left(\operatorname{Id}^{(h+k)}, G \circ F\right), \\
f \otimes g & \mapsto f \otimes g,
\end{aligned}
$$

soit un isomorphisme d'espaces vectoriels gradués;

(iii) l'application (13) soit un morphisme de modules de Yoneda, ce qui revient à dire que la structure de Yoneda de $\operatorname{Ext}_{\mathcal{P}}^{*}\left(\operatorname{Id}^{(h+k)}, G \circ F\right)$ est donnée sur les éléments $f \otimes g$ par la relation $(f \otimes g) \cdot e=f \otimes(g \cdot e)$.

On se fixe $f$ dans $\operatorname{Ext}_{\mathcal{P}}^{s}\left(\operatorname{Id}^{(k)}, G\right)$, qu'on peut supposer représenté par un facteur élémentaire $v$ de $\mathcal{R}_{G}^{s}$. Quitte à remplacer $\mathcal{R}_{G}^{\bullet}$ par la $S$-résolution réduite explicite $\mathcal{T}_{G}^{\bullet}$ de la proposition 3.5 (pour tous les indices $i \geq 1$ ), on peut supposer que le seul sommet de $\Gamma(v)$ égal à $S^{p^{k}}$ est $v$ lui-même.

Soit $I^{\bullet \bullet}$ une résolution injective de Cartan-Eilenberg de $\mathcal{R}_{G}^{\bullet} \circ F$. Son complexe total $\operatorname{Tot}\left(I^{\bullet \bullet}\right)$ est une résolution injective de $G \circ F$. Soit de même $J{ }^{\bullet \bullet}$ une résolution injective de Cartan-Eilenberg du complexe $\mathcal{R}_{G}^{\bullet}(v)$. Le facteur $v$ étant en degré $s$, la première colonne non nulle de $J^{\bullet \bullet}$ est d'abscisse $s$. L'inclusion $\mathcal{R}_{G}^{\bullet}(v) \circ F \rightarrow \mathcal{R}_{G}^{\bullet} \circ F$ induit un morphisme entre les résolutions de Cartan-Eilenberg, puis entre les complexes totaux associés, ainsi qu'entre les mêmes complexes auxquels on a appliqué $\operatorname{Hom}_{\mathcal{P}}\left(\operatorname{Id}^{(h+k)},-\right)$, et enfin entre la cohomologie de ces complexes:

$$
\mathrm{H}^{*}\left(\operatorname{Hom}_{\mathcal{P}}\left(\operatorname{Id}^{(h+k)}, \operatorname{Tot}\left(J^{\bullet \bullet}\right)\right)\right) \rightarrow \mathrm{H}^{*}\left(\operatorname{Hom}_{\mathcal{P}}\left(\operatorname{Id}^{(h+k)}, \operatorname{Tot}\left(I^{\bullet \bullet}\right)\right)\right) .
$$

L'argument du théorème 5.1 montre que le morphisme (14) est une injection, car $\mathcal{R}_{G}^{\bullet}(v) \rightarrow \mathcal{R}_{G}^{\bullet}$ induit une injection en rang 1 sur les premières suites spectrales d'hypercohomologie, et ces suites spectrales dégénèrent au rang 1 .

Soit $g$ un élément de $\operatorname{Ext}_{\mathcal{P}}^{t}\left(\operatorname{Id}^{(h+k)}, S^{p^{k}} \circ F\right)$. Puisque $J^{s, \bullet}$ est une résolution injective de $v \circ F=S^{p^{k}} \circ F$, l'extension $g$ est représentée par un morphisme $\widetilde{g}: \operatorname{Id}^{(h+k)} \rightarrow J^{s, t}$ tel que la composition

$$
\mathrm{Id}^{(h+k)} \stackrel{\widetilde{g}}{\longrightarrow} J^{s, t} \stackrel{d^{v}}{\longrightarrow} J^{s, t+1}
$$

soit nulle. Notons $\left(\mathrm{Fil}^{u}\right)$ la filtration $\operatorname{de} \operatorname{Hom}_{\mathcal{P}}\left(\operatorname{Id}^{(h+k)}, I^{\bullet \bullet}\right)$ définie par

$$
\mathrm{Fil}^{u}=\sum_{u^{\prime} \geq u} \operatorname{Hom}_{\mathcal{P}}\left(\operatorname{Id}^{(h+k)}, I^{u^{\prime}, \bullet}\right)
$$


et $\mathrm{Fil}^{u} \mathrm{H}$ la filtration de $\mathrm{H}^{*}\left(\operatorname{Hom}_{\mathcal{P}}\left(\operatorname{Id}^{(h+k)}, \operatorname{Tot}\left(I^{\bullet \bullet}\right)\right)\right)$ associée. On note de la même manière $\left(\mathrm{Fil}_{\Gamma}^{u}\right)$ et $\left(\mathrm{Fil}_{\Gamma}^{u} \mathrm{H}\right)$ les filtrations similaires pour le bicomplexe $J^{\bullet \bullet}$. D'après la proposition 3.5 , le seul facteur non égal à un produit tensoriel non trivial de $\mathcal{R}_{G}^{\bullet}(v)$ est $v$ lui-même : la suite spectrale $\mathbf{I}\left(\operatorname{Id}^{(h+k)}, \mathcal{R}_{G}^{\bullet}(v) \circ F\right)$ est nulle sur toutes les colonnes autres que celle d'abscisse $s$, égale à $\operatorname{Ext}_{\mathcal{P}}^{*}\left(\mathrm{Id}^{(h+k)}, S^{p^{k}} \circ F\right)$. Ainsi, la suite spectrale dégénère au rang 1 , et l'élément $g$, qui est non nul dans le tableau $\mathbf{I}_{1}^{s, t}\left(\operatorname{Id}^{(h+k)}, \mathcal{R}_{G}^{\bullet}(v) \circ F\right)$, est encore non nul dans l'aboutissement. Il définit donc un élément (encore noté $g$ ) de $\mathrm{Fil}_{\Gamma}^{s} \mathrm{H} / \mathrm{Fil}_{\Gamma}^{s+1} \mathrm{H}$, en degré total $s+t$. Mais comme la suite spectrale est nulle en abscisses autres que $s$, chacun des quotients $\mathrm{Fil}_{\Gamma}^{u} \mathrm{H} / \mathrm{Fil}_{\Gamma}^{u+1} \mathrm{H}$ est nul pour $u \neq s$. Ainsi, $\mathrm{Fil}_{\Gamma}^{s+1} \mathrm{H}$ est nul, et $\mathrm{Fil}_{\Gamma}^{s} \mathrm{H}$ est égale à la cohomologie totale. Par conséquent, $g$ définit sans ambiguité un élément non nul $g^{\prime}$ de $\mathrm{H}^{s+t}\left(\operatorname{Hom}_{\mathcal{P}}\left(\operatorname{Id}^{(h+k)}, \operatorname{Tot}\left(J^{\bullet \bullet}\right)\right)\right)$.

L'élément $f \otimes g$ dans $\mathrm{H}^{h+t}\left(\operatorname{Hom}_{\mathcal{P}}\left(\operatorname{Id}^{(h+k)}, \operatorname{Tot}\left(I^{\bullet \bullet}\right)\right)\right)$, c'est-à-dire dans $\operatorname{Ext}_{\mathcal{P}}^{*}\left(\operatorname{Id}^{(h+k)}, G \circ F\right)$, est alors par définition l'image de $g^{\prime}$ par (14).

Le morphisme

$$
\begin{aligned}
\operatorname{Ext}_{\mathcal{P}}^{*}\left(\operatorname{Id}^{(k)}, G\right) \otimes \operatorname{Ext}_{\mathcal{P}}^{*}\left(\operatorname{Id}^{(h+k)}, S^{p^{k}} \circ F\right) & \rightarrow \operatorname{Ext}_{\mathcal{P}}^{*}\left(\operatorname{Id}^{(h+k)}, G \circ F\right), \\
f \otimes g & \mapsto f \otimes g,
\end{aligned}
$$

est clairement bilinéaire. On veut montrer qu'il s'agit en fait d'un isomorphisme. En raison du théorème 5.1, les dimensions (degré par degré) sont les mêmes pour l'espace source et l'espace but. Ainsi, il suffit de montrer la surjectivité. Soit $\widetilde{g}^{\prime}: \operatorname{Id}^{(h+k)} \rightarrow \operatorname{Tot}\left(J^{\bullet \bullet}\right)$ un représentant de $g^{\prime}$. Alors, l'image de $\widetilde{g}^{\prime}$ par le morphisme $\operatorname{Tot}\left(J^{\bullet \bullet}\right) \rightarrow \operatorname{Tot}\left(I^{\bullet \bullet}\right)$ est un représentant de $f \otimes g$. On en déduit que $f \otimes g$ est un élément du $s$-ième terme $\mathrm{Fil}^{s} \mathrm{H}$ de la filtration de $\operatorname{Ext}_{\mathcal{P}}^{*}\left(\mathrm{Id}^{(h+k)}, G \circ F\right)$. De plus, son image dans $\mathrm{Fil}^{s} \mathrm{H} / \mathrm{Fil}^{s+1} \mathrm{H}$ est exactement égal à l'élément $f \otimes g$ de $\mathbf{I}_{\infty}\left(\operatorname{Id}^{(h+k)}, \mathcal{R}_{G}^{\bullet} \circ F\right)$. Il en résulte que l'égalité $\mathrm{Fil}{ }^{s} \mathrm{H} / \mathrm{Fil}^{s+1} \mathrm{H} \rightarrow \mathrm{Fil}^{s} \mathrm{H} / \mathrm{Fil}^{s+1} \mathrm{H}$ se factorise par le sous-espace gradué

$$
\operatorname{Ext}_{\mathcal{P}}^{s}\left(\operatorname{Id}^{(k)}, G\right) \otimes \operatorname{Ext}^{*}\left(\operatorname{Id}^{(h+k)}, S^{p^{k}} \circ F\right),
$$

de $\operatorname{Ext}_{\mathcal{P}}^{*}\left(\operatorname{Id}^{(h+k)}, G \circ F\right)$ la graduation étant donnée par $*+s$, où la notation $\operatorname{Ext}_{\mathcal{P}}^{s}\left(\operatorname{Id}^{(k)}, G\right) \otimes \operatorname{Ext}_{\mathcal{P}}^{*}\left(\operatorname{Id}^{(h+k)}, S^{p^{k}} \circ F\right)$ désigne le sous-espace engendré par tous les éléments $f \otimes g$, pour $f \in \operatorname{Ext}_{\mathcal{P}}^{s}\left(\operatorname{Id}^{(k)}, G\right)$ et $g \in \operatorname{Ext}_{\mathcal{P}}^{*}\left(\operatorname{Id}^{(h+k)}, S^{p^{k}} \circ F\right)$. Par conséquent, pour tout $s$,

$$
\operatorname{dim} \operatorname{Ext}_{\mathcal{P}}^{s}\left(\operatorname{Id}^{(k)}, G\right) \otimes \operatorname{Ext}_{\mathcal{P}}^{*}\left(\operatorname{Id}^{(h+k)}, S^{p^{k}} \circ F\right) \geq \operatorname{dim} \mathrm{Fil}^{s} \mathrm{H} / \mathrm{Fil}^{s+1} \mathrm{H},
$$

puis

$$
\operatorname{dim} \operatorname{Ext}_{\mathcal{P}}^{*}\left(\operatorname{Id}^{(k)}, G\right) \otimes \operatorname{Ext}_{\mathcal{P}}^{*}\left(\operatorname{Id}^{(h+k)}, S^{p^{k}} \circ F\right) \geq \operatorname{dim} \operatorname{Ext}_{\mathcal{P}}^{*}\left(\operatorname{Id}^{(h+k)}, G \circ F\right) .
$$

Comme le premier est un sous-espace du deuxième, on en déduit l'égalité des dimensions, et donc des espaces : $f \otimes g \mapsto f \otimes g$ est surjective. 
Il reste à montrer que c'est un isomorphisme de modules. Avec $f$ et $g$ comme précédemment, il faut montrer, pour tout $e \in \operatorname{Ext}_{\mathcal{P}}^{i}\left(\operatorname{Id}^{(h+k)}, \operatorname{Id}^{(h+k)}\right)$, $(f \otimes g) \cdot e=f \otimes(g \cdot e)$. Soit

$$
0 \rightarrow \mathrm{Id}^{(h+k)} \rightarrow U_{1} \rightarrow \ldots \rightarrow U_{i} \rightarrow \mathrm{Id}^{(h+k)} \rightarrow 0
$$

un représentant "à la Yoneda" de l'extension $e$. On obtient un représentant $\widetilde{h}^{\prime}: \operatorname{Id}^{(h+k)} \rightarrow \operatorname{Tot}\left(J^{\bullet \bullet}\right)$ du produit $g^{\prime} \cdot e$ dans la cohomologie totale $\operatorname{Ext}_{\mathcal{P}}^{*}\left(\operatorname{Id}^{(h+k)}, \mathcal{R}_{G}^{\bullet}(v) \circ F\right)$ en construisant un morphisme de complexes entre le complexe acyclique (15) et le complexe injectif $\operatorname{Tot}\left(J^{\bullet \bullet}\right)$ :

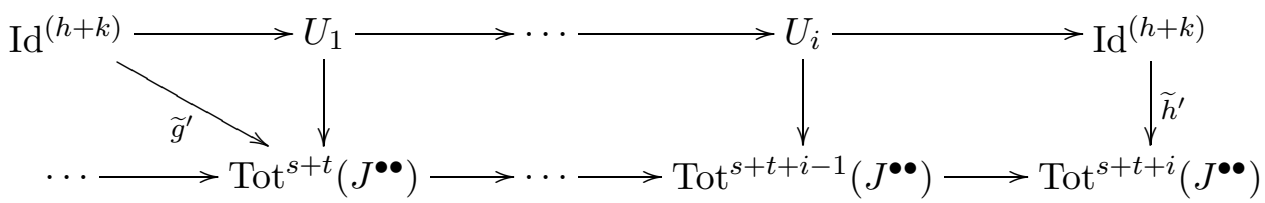

Par composition de $\widetilde{h}^{\prime}$ avec la projection $\operatorname{Tot}^{s+t+i}\left(J^{\bullet \bullet}\right) \rightarrow J^{s, t+i}$ on obtient un représentant $h: \operatorname{Id}^{(h+k)} \rightarrow J^{s, t+i}$ de $g \cdot e$, dans la résolution $v \circ F \rightarrow J^{s, \bullet}$ de $v \circ F=S^{p^{k}} \circ F$. Ainsi, l'image de la classe de $\widetilde{h}^{\prime}$ par le morphisme

$$
\operatorname{Ext}_{\mathcal{P}}^{*}\left(\operatorname{Id}^{(h+k)}, \mathcal{R}_{G}^{\bullet}(v) \circ F\right) \rightarrow \operatorname{Ext}_{\mathcal{P}}^{*}\left(\operatorname{Id}^{(h+k)}, \mathcal{R}_{G}^{\bullet} \circ F\right)
$$

est $f \otimes(g \cdot e)$. D'un autre côté, comme (16) est un morphisme de modules de Yoneda, l'image de la classe de $\widetilde{h}^{\prime}$ par $(16)$ est aussi égale à $(f \otimes g) \cdot e$.

Le fait que (12) est un isomorphisme de modules se démontre de la même manière.

Remarque 5.3. D'après le théorème 1.7, on déduit des formules analogues à celles du théorème $\mathrm{A}$ pour les modules $\operatorname{Ext}_{\mathcal{P}}^{*}\left(\operatorname{Id}^{(h+k+r)},(G \circ F)^{(r)}\right)$ et $\operatorname{Ext}_{\mathcal{F}}^{*}(\mathrm{Id}, G \circ F)$.

6. $\operatorname{Ext}_{\mathcal{P}}^{*}\left(\operatorname{Id}^{(h+k)}, S^{p^{k}} \circ S^{p^{h}}\right)$ en caractéristique 2. Dans la fin de cet article, nous proposons une alternative au calcul de $\operatorname{Ext}_{\mathcal{F}}^{*}\left(\mathrm{Id}, S^{2^{k}} \circ S^{2^{h}}\right)$ en caractéristique $p=2$, contournant les calculs de [16]. La méthode repose sur le même principe général, à savoir l'emploi des suites spectrales d'hypercohomologie des complexes $\mathcal{S}_{2^{k}}^{\bullet} \circ S^{2^{h}}$ obtenus en précomposant le complexe $\mathcal{S}_{2^{k}}^{\bullet}$ (égal à la partie de degré $2^{k}$ de la construction Cobar réduite du foncteur en algèbres de Hopf $S^{*}$ ) par le foncteur $S^{2^{h}}$. Le théorème 5.1 nous permet beaucoup plus facilement que dans [16] de déterminer les différentielles de ces suites spectrales. En particulier, nous n'avons pas besoin de connaître les groupes d'extensions $\operatorname{Ext}_{\mathcal{F}}^{*}\left(\mathrm{Id}, S^{n} \circ I\right)$, le foncteur $I$ étant l'injectif standard de $\mathcal{F}$ défini par $I(V)=\mathbb{F}_{2}^{V^{*}}$; cela nous évite le passage par la catégorie $\mathcal{F}$, passage indispensable lorsqu'on considère le foncteur $I$, puisque ce foncteur n'est pas dans l'image de $\mathcal{P}$ par le foncteur oubli. Le seul antécédent que nous utilisons est le calcul, ne présentant pas de difficulté technique, du mo- 
dule $\operatorname{Ext}_{\mathcal{P}}^{*}\left(\operatorname{Id}^{(h+1)}, S^{2} \circ S^{2^{h}}\right)($ cf. [15]), et plus spécifiquement le fait que sa structure de module de Yoneda est triviale.

Dans tout ce qui suit, $p=2$. On rappelle qu'on considère dans $[15,16]$ des complexes $\mathcal{S}_{n}^{\bullet}$ définis comme la partie de degré $n$ de la construction Cobar réduite du foncteur en algèbres de Hopf $S^{*}$ :

$$
\mathcal{S}_{n}^{\bullet}: S^{n} \rightarrow \bigoplus_{\substack{i_{1}+i_{2}=n \\ i_{1}, i_{2}>0}} S^{i_{1}} \otimes S^{i_{2}} \rightarrow \bigoplus_{\substack{i_{1}+i_{2}+i_{3}=n \\ i_{1}, i_{2}, i_{3}>0}} S^{i_{1}} \otimes S^{i_{2}} \otimes S^{i_{3}} \rightarrow \ldots \rightarrow\left(S^{1}\right)^{\otimes n}
$$

On connaît leur cohomologie (cf. [9, Theorem I.4.27] ou [2]) :

$$
\mathrm{H}^{t}\left(\mathcal{S}_{n}^{\bullet}\right)=\bigoplus_{\substack{\sum_{l \geq 0} i_{l}=t \\ \sum_{l \geq 0} i_{l} 2^{2}=n}} \bigotimes_{l \geq 0} S^{i_{l}(l)}
$$

Supposons maintenant que $n$ est une puissance de 2 , disons $n=2^{k}$. Grâce au théorème de Pirashvili, on détermine la seconde suite spectrale du complexe composé $\mathcal{S}_{2^{k}}^{\bullet}$ au rang 2 : elle est nulle sur toutes ses lignes, sauf ses lignes d'ordonnée $2^{l}, 0 \leq l \leq k$, sur lesquelles elle est donnée par

$$
\mathbf{I I}_{2}^{*, 2^{l}}\left(\operatorname{Id}^{(h+k+r)},\left(\mathcal{S}_{2^{k}}^{\bullet} \circ S^{2^{h}}\right)^{(r)}\right)=\operatorname{Ext}_{\mathcal{P}}^{*}\left(\operatorname{Id}^{(h+k+r)},\left(S^{2^{l}} \circ S^{2^{h}}\right)^{(k+r-l)}\right) .
$$

Le morphisme de complexes $\left(\mathcal{S}_{2^{k-1}}^{\bullet}\right)^{(1)} \rightarrow \mathcal{S}_{2^{k}}^{\bullet}$ défini par le morphisme de Frobenius induit un morphisme sur les suites spectrales

$$
\mathbf{I I}\left(\operatorname{Id}^{(h+k+r)},\left(\mathcal{S}_{2^{k-1}}^{\bullet} \circ S^{2^{h}}\right)^{(r+1)}\right) \rightarrow \mathbf{I I}\left(\operatorname{Id}^{(h+k+r)},\left(\mathcal{S}_{2^{k}}^{\bullet} \circ S^{2^{h}}\right)^{(r)}\right),
$$

consistant au rang 2 en l'inclusion des $2^{k-1}$ premières lignes. Ainsi, seule la ligne $2^{k}$ de la suite spectrale $\mathbf{I I}_{2}\left(\operatorname{Id}^{(h+k+r)},\left(\mathcal{S}_{2^{k}}^{\bullet} \circ S^{2^{h}}\right)^{(r)}\right)$ n'est pas dans l'image de ce morphisme. Comme les différentielles non nulles issues de cette ligne sont au moins de degré $2^{k-1}+1$, on en déduit notamment que

$$
\mathbf{I I}_{2^{k-1}+1}\left(\operatorname{Id}^{(h+k+r)},\left(\mathcal{S}_{2^{k-1}}^{\bullet} \circ S^{2^{h}}\right)^{(r+1)}\right) \rightarrow \mathbf{I I}_{2^{k-1}+1}\left(\operatorname{Id}^{(h+k)},\left(\mathcal{S}_{2^{k}}^{\bullet} \circ S^{2^{h}}\right)^{(r)}\right)
$$

est l'inclusion des $2^{k-1}$ premières lignes. Par conséquent, toutes les différentielles entre les lignes $0, \ldots, 2^{k-1}$ sont les mêmes dans les deux suites spectrales. Pour déterminer toutes les différentielles de toutes les suites spectrales $\mathbf{I I}\left(\operatorname{Id}^{(h+k+r)},\left(\mathcal{S}_{2^{k}}^{\bullet} \circ S^{2^{h}}\right)^{(r)}\right), k \geq 0$, il suffit donc de déterminer pour tout $k \geq 0$ les différentielles issues de la ligne $2^{k}$ de la suite spectrale II $\left(\operatorname{Id}^{(h+\bar{k}+r)},\left(\mathcal{S}_{2^{k}}^{\bullet} \circ S^{2^{h}}\right)^{(r)}\right)$. La description de ces différentielles fait l'objet de la proposition suivante.

Proposition 6.1. Les différentielles issues de la ligne $2^{k}$ de la suite spectrale $\mathbf{I I}\left(\mathrm{Id}^{(h+k+r)},\left(\mathcal{S}_{2^{k}}^{\bullet} \circ S^{2^{h}}\right)^{(r)}\right)$ sont

- nulles si le quotient de la division euclidienne de l'abscisse de leur but par $2^{h+k}$ est pair; 
- surjectives si le quotient de la division euclidienne de l'abscisse de leur but par $2^{h+k}$ est impair.

Suivant [16], on en déduit l'expression du module $\operatorname{Ext}_{\mathcal{P}}^{*}\left(\operatorname{Id}^{(h+k)}, S^{2^{k}} \circ S^{2^{h}}\right)$.

THÉORÈmE 6.2. La structure de module de $\operatorname{Ext}_{\mathcal{P}}^{*}\left(\operatorname{Id}^{(h+k)}, S^{2^{k}} \circ S^{2^{h}}\right)$ est triviale. Sa série de Poincaré $\varphi_{h, k}$ est donnée par

$$
\begin{aligned}
\varphi_{h, k}(t) & =\sum_{i \geq 0} t^{i} \cdot \operatorname{dim} \operatorname{Ext}_{\mathcal{P}}^{i}\left(\operatorname{Id}^{(h+k)}, S^{2^{k}} \circ S^{2^{h}}\right) \\
& =\frac{\prod_{i=1}^{h+k}\left(1-t^{2^{i}-1}\right)}{\prod_{i=1}^{h}\left(1-t^{2^{i}-1}\right) \prod_{i=1}^{k}\left(1-t^{2^{i}-1}\right)} .
\end{aligned}
$$

En utilisant le théorème 1.7, on obtient également une description des séries de Poincaré de $\operatorname{Ext}_{\mathcal{P}}^{*}\left(\operatorname{Id}^{(h+k+r)},\left(S^{2^{k}} \circ S^{2^{h}}\right)^{(r)}\right)$ et de $\operatorname{Ext}_{\mathcal{F}}^{*}\left(\operatorname{Id}, S^{2^{k}} \circ S^{2^{h}}\right)$, ainsi que leur structure de module.

La démonstration de la proposition 6.1 constitue le coeur de [16]. La preuve présentée dans [16] est par endroits délicate et calculatoire. On présente ici une preuve évitant ces calculs, basée sur le lemme suivant.

LEMme 6.3. La structure de module de Yoneda de $\operatorname{Ext}_{\mathcal{P}}^{*}\left(\operatorname{Id}^{(h+k)}, S^{2^{k}} \circ S^{2^{h}}\right)$ est triviale.

Démonstration. Comme $S^{2^{k}} \circ S^{2^{h}}$ est un facteur direct de $\left(S^{2}\right)^{\circ(h+k)}$, il suffit de montrer que pour tout $l$, la structure de module de Yoneda de $\operatorname{Ext}_{\mathcal{P}}^{*}\left(\operatorname{Id}^{(h+k)},\left(S^{2}\right)^{\circ l}\right)$ est triviale, ce qu'on montre par récurrence sur $l$. Le point de départ est le calcul effectué dans [15] de $\operatorname{Ext}_{\mathcal{P}}^{*}\left(\operatorname{Id}^{(k+1)}, S^{2^{k}} \circ S^{2}\right)$. On y montre notamment que sa structure de module est triviale. Cela implique que le résultat est vrai pour $l=1,2$.

Supposons qu'il est vrai pour $l$. Les théorèmes 5.1 et 5.2 fournissent alors un isomorphisme de modules de Yoneda

$$
\begin{aligned}
\operatorname{Ext}_{\mathcal{P}}^{*} & \left(\operatorname{Id}^{(l+1)},\left(S^{2}\right)^{\circ(l+1)}\right) \\
\quad & \cong \operatorname{Ext}_{\mathcal{P}}^{*}\left(\operatorname{Id}^{(1)}, S^{2}\right) \otimes \operatorname{Ext}_{\mathcal{P}}^{*}\left(\operatorname{Id}^{(l)},\left(S^{2}\right)^{\circ l}\right) \otimes \operatorname{Ext}_{\mathcal{P}}^{*}\left(\operatorname{Id}^{(l+1)}, S^{2^{l}} \circ S^{2}\right) .
\end{aligned}
$$

L'action de Yoneda sur le produit tensoriel est l'action sur le troisième facteur. Cette action est nulle d'après [15].

Démonstration de la proposition 6.1. On note pour simplifier I et II les deux suites spectrales d'hypercohomologie $\mathbf{I}\left(\operatorname{Id}^{(h+k+r)},\left(\mathcal{S}_{2^{k}}^{\bullet} \circ S^{2^{h}}\right)^{(r)}\right)$ et $\mathbf{I I}\left(\operatorname{Id}^{(h+k+r)},\left(\mathcal{S}_{2^{\bullet}}^{\bullet} \circ S^{2^{h}}\right)^{(r)}\right)$.

Soient $l<k$ et $t$ un entier dont le quotient par la division euclidienne par $2^{h+k}$ est pair. Soit $d: \mathbf{I I}^{s, 2^{k}} \rightarrow \mathbf{I I}^{t, 2^{l}}$ la différentielle de rang $2^{k}-2^{l}+1$ issue de la ligne $2^{k}$ et de but $\left(t, 2^{l}\right)$. La structure de module de Yoneda de la suite spectrale II donne un carré commutatif 


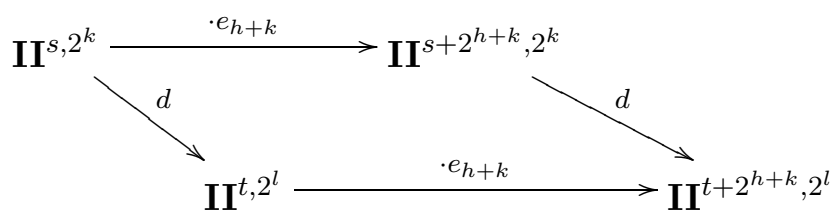

D'après le lemme 6.3 et les théorèmes 1.7 et 1.3, l'action de $e_{h+k}$ donne un isomorphisme entre $\mathbf{I I}_{2}^{t, 2^{l}}$ et $\mathbf{I I}_{2}^{t+2^{h+k}, 2^{l}}$. Par un argument de récurrence sur le rang de la différentielle $d$, cela reste un isomorphisme au rang considéré dans le diagramme ci-dessus : le morphisme du bas est un isomorphisme. Par le lemme 6.3, le morphisme du haut est nul. Ainsi, pour que le carré soit commutatif, la différentielle de gauche doit être nulle.

Soit maintenant $t$ un entier dont le quotient par la division euclidienne par $2^{h+k}$ est impair, et considérons l'action de $e_{h+k}$ dans II. Comme précédemment, elle réalise une bijection

$$
\mathbf{I I}^{t-2^{h+k}, 2^{l}} \stackrel{\simeq}{\longrightarrow} \mathbf{I I}^{t, 2^{l}}
$$

en rang $2^{k-1}+1$. Supposons que $\mathbf{I I}_{\infty}^{t, 2^{l}}$ est non nul. Par l'argument précédent, $\mathbf{I I}_{2^{k-1}+1}^{t-2^{h+k}, 2^{l}}$ est alors égal à $\mathbf{I I}_{\infty}^{t-2^{h+k}, 2^{l}}$. Ainsi, l'isomorphisme (17) induit une action non nulle de $e_{h+k}$ dans l'aboutissement. Comme l'aboutissement est égal à $\operatorname{Ext}_{\mathcal{P}}^{*-1}\left(\operatorname{Id}^{(h+k)}, S^{2^{k}} \circ S^{2^{h}}\right)$ en tant que module, cela contredit le lemme 6.3. Par conséquent, $\mathbf{I I}_{\infty}^{t, 2^{l}}=0$. Comme la seule différentielle non nulle de rang supérieur ou égal à $2^{k-1}+1$ de source ou de but $\mathbf{I I}^{t, 2^{l}}$ est celle issue de la ligne $2^{k}$, celle-ci doit être surjective.

Remarque 6.4. Le seul endroit où on utilise de manière cruciale le théorème 1.6 est lors du calcul de $\operatorname{Ext}_{\mathcal{P}}^{*}\left(\operatorname{Id}^{(h+1)}, S^{2^{h}} \circ S^{2}\right)$ (cf. [15]). En inversant le sens des compositions, il existe toujours un endroit dans la preuve où on utilise le théorème 1.6. Cette présentation semble la plus économique de ce point de vue.

Remarque 6.5. Cette démonstration ne s'adapte pas facilement au cas où $p$ est impair, car dans ce cas, les structures de modules de Yoneda de $\operatorname{Ext}_{\mathcal{P}}^{*}\left(\operatorname{Id}^{(h+1)}, S^{p} \circ S^{p^{h}}\right)$ et de $\operatorname{Ext}_{\mathcal{P}}^{*}\left(\operatorname{Id}^{(h+1)}, S^{p^{h}} \circ S^{p}\right)$ sont non triviales [15].

Notons enfin que le théorème $\mathrm{B}$ se déduit des théorèmes 5.1, 5.2, 6.2 et le lemme 6.3 par une récurrence immédiate sur $l$.

7. Quelques exemples. On termine en donnant quelques exemples de foncteurs vérifiant les hypothèses du théorème 5.1, en caractéristique 2 . On notera qu'à condition de savoir calculer Ext*(Id, $F)$ pour chacun de ces foncteurs $F$ (ce qui est le cas dans les exemples ci-dessous), on est capable d'en déduire les modules $\operatorname{Ext}\left(\operatorname{Id}, F_{n} \circ \ldots \circ F_{1}\right)$ pour toute composition de tels foncteurs $F_{1}, \ldots, F_{n}$. 
Dans cette section, $p=2$. Soit $(\mathcal{H})$ la propriété requise pour les foncteurs $F$ et $G$ dans le théorème 5.1 : un foncteur $F \in \mathcal{P}_{p^{h}}$ vérifie la propriété $(\mathcal{H})$ si et seulement si $\operatorname{Ext}_{\mathcal{P}}^{*}\left(\operatorname{Id}^{(h)}, F\right)$ est un module trivial.

Les premiers exemples sont bien-entendu les puissances symétriques, extérieures et divisées, ainsi que toute composition de puissances symétriques, extérieures ou divisées. Cela entraîne que la propriété $(\mathcal{H})$ est stable par composition (ce n'est pas vrai en caractéristique $p>2$ ).

On donne quelques exemples supplémentaires dans le contexte des foncteurs de Schur et des foncteurs simples. Pour les définitions et notations, on renvoie à [12, Appendice $\mathrm{A}]$.

Proposition 7.1. Les foncteurs de Schur $W_{(n, 1)^{\prime}}$, noyaux de $\Lambda^{n} \otimes \Lambda^{1} \rightarrow$ $\Lambda^{n+1}$, vérifient la propriété $(\mathcal{H})$.

Démonstration. Si $n+1$ n'est pas une puissance de $2, \operatorname{Ext}_{\mathcal{F}}^{*}\left(\operatorname{Id}, W_{(n, 1)^{\prime}}\right)$ est nul d'après le théorème 1.7. Sinon, la suite exacte courte

$$
0 \rightarrow W_{\left(2^{h}-1,1\right)^{\prime}} \rightarrow \Lambda^{2^{h}-1} \otimes \Lambda^{1} \rightarrow \Lambda^{2^{h}} \rightarrow 0
$$

et l'expression de $\operatorname{Ext}_{\mathcal{P}}^{*}\left(\operatorname{Id}^{(h)}, \Lambda^{2^{h}}\right)$ montrent que

$$
\operatorname{Ext}_{\mathcal{P}}^{i}\left(\operatorname{Id}^{(h)}, W_{\left(2^{h}-1,1\right)^{\prime}}\right)= \begin{cases}\mathbb{F}_{2} & \text { si } i=2^{h} \\ 0 & \text { sinon. }\end{cases}
$$

Seul un terme est non nul : la structure de module est forcément triviale.

Proposition 7.2. Le foncteur simple $S_{(3,1)^{\prime}}$ vérifie la propriété $(\mathcal{H})$.

Démonstration. En étudiant la structure du produit tensoriel $\Lambda^{2} \otimes \Lambda^{2}$, on montre que $\Lambda^{2} \circ \Lambda^{2}$ est la somme directe de $\Lambda^{4}$ et du foncteur simple $S_{(3,1)^{\prime}}$. Comme $\Lambda^{2} \circ \Lambda^{2}$ vérifie la propriété $(\mathcal{H})$, le foncteur simple $S_{(3,1)^{\prime}}$ aussi.

Proposition 7.3. Le foncteur simple $S_{(7,1)^{\prime}}$ vérifie la propriété $(\mathcal{H})$.

Démonstration. On commence par calculer $\operatorname{Ext}_{\mathcal{P}}^{*}\left(\operatorname{Id}^{(3)}, S_{(7,1)^{\prime}}\right)$ en tant qu'espace vectoriel gradué, en utilisant la suite exacte courte

$$
0 \rightarrow \Lambda^{8} \rightarrow W_{(7,1)^{\prime}} \rightarrow S_{(7,1)^{\prime}} \rightarrow 0 .
$$

L'espace vectoriel gradué $\operatorname{Ext}_{\mathcal{P}}^{*}\left(\operatorname{Id}^{(3)}, W_{(7,1)^{\prime}}\right)$ est de dimension 1 en degré 8 et de dimension 0 sinon (proposition 7.1 ), alors que $\operatorname{Ext}_{\mathcal{P}}^{*}\left(\operatorname{Id}^{(3)}, \Lambda^{8}\right)$ est de dimension 1 en degré 7 , et de dimension 0 sinon (cf. [6]). Ainsi, les morphismes

$$
\operatorname{Ext}_{\mathcal{P}}^{i}\left(\operatorname{Id}^{(3)}, \Lambda^{8}\right) \rightarrow \operatorname{Ext}_{\mathcal{P}}^{i}\left(\operatorname{Id}^{(3)}, W_{(7,1)^{\prime}}\right)
$$

apparaissant dans la suite exacte longue associée à la suite exacte courte (18) sont toujours nuls. Cette suite exacte longue se scinde donc en suites 
exactes courtes

$$
0 \rightarrow \operatorname{Ext}_{\mathcal{P}}^{i}\left(\operatorname{Id}^{(3)}, W_{(7,1)^{\prime}}\right) \rightarrow \operatorname{Ext}_{\mathcal{P}}^{i}\left(\operatorname{Id}^{(3)}, S_{(7,1)^{\prime}}\right) \rightarrow \operatorname{Ext}_{\mathcal{P}}^{i+1}\left(\operatorname{Id}^{(3)}, \Lambda^{8}\right) \rightarrow 0
$$

et par conséquent, on obtient un isomorphisme d'espaces vectoriels gradués

$$
\operatorname{Ext}_{\mathcal{P}}^{*}\left(\operatorname{Id}^{(3)}, S_{(7,1)^{\prime}}\right) \cong \operatorname{Ext}_{\mathcal{P}}^{*}\left(\operatorname{Id}^{(3)}, W_{(7,1)^{\prime}}\right) \oplus \operatorname{Ext}_{\mathcal{P}}^{*+1}\left(\operatorname{Id}^{(3)}, \Lambda^{8}\right) .
$$

L'espace $\operatorname{Ext}_{\mathcal{P}}^{*}\left(\operatorname{Id}^{(3)}, S_{(7,1)^{\prime}}\right)$ est donc décrit par

$$
\operatorname{Ext}_{\mathcal{P}}^{i}\left(\operatorname{Id}^{(3)}, S_{(7,1)^{\prime}}\right)= \begin{cases}\mathbb{F}_{2} & \text { si } i=2^{h}-2,2^{h} \\ 0 & \text { sinon. }\end{cases}
$$

Cette description ne suffit pas à conclure quant à la trivialité de la struc-

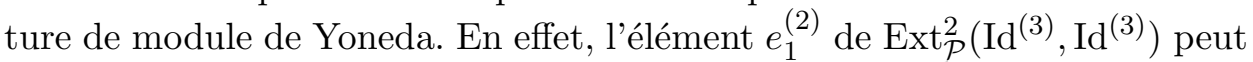
donner une action non nulle envoyant le terme de degré $2^{h}-2$ bijectivement sur le terme de degré $2^{h}$.

La démonstration de la trivialité de la structure de module est basée sur l'étude de la série de Jordan-Hölder de $\Lambda^{6} \otimes \Lambda^{2}$. Pour cela, on s'aide de résultats issus de la théorie des représentations du groupe symétrique [8]. Le foncteur $\Lambda^{6} \otimes \Lambda^{2}$ admet une filtration dont les quotients sont $\Lambda^{8}, W_{(7,1)^{\prime}}$ et $W_{(6,2)^{\prime}}$ (cf. coefficients de Littlewood-Richardson [8]). Des résulats de représentation du groupe symétrique donnent les quotients simples de la série de Jordan-Hölder des foncteurs de Schur $W_{(7,1)^{\prime}}$ et $W_{(6,2)^{\prime}}$. En particulier, on en déduit que la série de Jordan-Hölder de $\Lambda^{6} \otimes \Lambda^{2}$ a deux quotients égaux à $\Lambda^{8}$. L'un d'eux est un quotient de $\Lambda^{6} \otimes \Lambda^{2}$, puisqu'il existe un morphisme surjectif $\Lambda^{6} \otimes \Lambda^{2} \rightarrow \Lambda^{8}$. L'autre est donc un sous-objet de $\Lambda^{6} \otimes \Lambda^{2}$, puisque $\Lambda^{6} \otimes \Lambda^{2}$ et $\Lambda^{8}$ sont autoduaux.

Le foncteur $W_{(7,1)^{\prime}}$ est un quotient de $\Lambda^{6} \otimes \Lambda^{2}$. En effet, $W_{(7,1)^{\prime}}$ est le noyau du morphisme $\Lambda^{7} \otimes \Lambda^{1} \rightarrow \Lambda^{8}$. Par exactitude de

$$
\Lambda^{6} \otimes \Lambda^{2} \rightarrow \Lambda^{7} \otimes \Lambda^{1} \rightarrow \Lambda^{8}
$$

on en déduit un morphisme surjectif $\Lambda^{6} \otimes \Lambda^{2} \rightarrow W_{(7,1)^{\prime}}$.

De plus, les foncteurs simples apparaissant comme quotients de la série de Jordan-Hölder de $W_{(7,1)^{\prime}}$ sont, d'après les tables de [8], $S_{(7,1)^{\prime}}$ et $\Lambda^{8}$. Comme $S_{(7,1)^{\prime}}$ est par définition un quotient de $W_{(7,1)^{\prime}}, \Lambda^{8}$ en est un sous-objet. Mais il n'en est pas un quotient. Ainsi, dans l'inclusion $W_{(7,1)^{\prime}} \rightarrow \Lambda^{6} \otimes \Lambda^{2}$, ce foncteur simple $\Lambda^{8}$ correspond au terme $\Lambda^{8}$ qui est sous-objet de $\Lambda^{6} \otimes \Lambda^{2}$, et non à celui qui est quotient.

Soit maintenant une filtration $0=F_{3} \subset F_{2} \subset F_{1} \subset F_{0}=\Lambda^{6} \otimes \Lambda^{2}$ de $\Lambda^{6} \otimes \Lambda^{2}$ dont les quotients sont, dans un certain ordre, $\Lambda^{8}, W_{(7,1)^{\prime}}$ et $W_{(6,2)^{\prime}}$. Puisque $W_{(7,1)^{\prime}}$ est un sous-objet, on peut s'arranger pour que ce soit le premier quotient de la filtration :

$$
W_{(7,1)^{\prime}} \cong F_{0} / F_{1}
$$


On peut affiner la filtration ci-dessus en intercalant des termes afin d'obtenir une série de Jordan-Hölder. Nous obtenons une série de composition

$$
\ldots \subset G_{2} \subset G_{1} \subset G_{0}=\Lambda^{6} \otimes \Lambda^{2},
$$

dont les deux premiers quotients $G_{0} / G_{1}$ et $G_{1} / G_{2}$ sont $S_{(7,1)^{\prime}}$ et $\Lambda^{8}$. Ce facteur simple $\Lambda^{8}$ correspond au facteur qui est sous-objet de $\Lambda^{6} \otimes \Lambda^{2}$. Cela signifie que le facteur $\Lambda^{8}$ correspondant au facteur sous-objet de $\Lambda^{6} \otimes \Lambda^{2}$ est un facteur de composition supérieur du noyau $K$ de la surjection $\Lambda^{6} \otimes \Lambda^{2} \rightarrow$ $S_{(7,1)^{\prime}}$. Il est donc facteur direct de $K$.

Soit $K^{\prime}$ le foncteur défini par l'égalité $K=\Lambda^{8} \oplus K^{\prime}$. On considère les deux suites spectrales I et II obtenues en appliquant $\operatorname{Hom}_{\mathcal{P}}\left(\operatorname{Id}^{(4)},-\right)$ à une résolution injective de Cartan-Eilenberg de la suite exacte courte

$$
0 \rightarrow \Lambda^{8} \oplus K^{\prime} \rightarrow \Lambda^{6} \otimes \Lambda^{2} \rightarrow S_{(7,1)^{\prime}} \rightarrow 0 .
$$

La seconde suite spectrale II est nulle, par exactitude de la suite. La première suite spectrale $\mathbf{I}$ converge donc vers 0 . D'après le théorème de Pirashvili, elle est nulle sur sa colonne 2 (on a placé $K$ en degré 1 ). Elle n'a donc que deux colonnes non nulles :

$$
\begin{aligned}
& \mathbf{I}_{2}^{1, *}=\operatorname{Ext}_{\mathcal{P}}^{*}\left(\operatorname{Id}^{(4)}, S_{(7,1)^{\prime}}\right), \\
& \mathbf{I}_{2}^{3, *}=\operatorname{Ext}_{\mathcal{P}}^{*}\left(\operatorname{Id}^{(4)}, \Lambda^{8}\right) \oplus \operatorname{Ext}_{\mathcal{P}}^{*}\left(\operatorname{Id}^{(4)}, K^{\prime}\right) .
\end{aligned}
$$

Par conséquent, la différentielle de rang 2 est une bijection entre les colonnes 1 et 3. Cette différentielle fournit un isomorphisme de modules de Yoneda

$$
\operatorname{Ext}_{\mathcal{P}}^{*+1}\left(\operatorname{Id}^{(4)}, S_{(7,1)^{\prime}}\right)=\operatorname{Ext}_{\mathcal{P}}^{*}\left(\operatorname{Id}^{(4)}, \Lambda^{8}\right) \oplus \operatorname{Ext}_{\mathcal{P}}^{*}\left(\operatorname{Id}^{(4)}, K^{\prime}\right) .
$$

La structure de module de Yoneda de $\operatorname{Ext}_{\mathcal{P}}^{*}\left(\operatorname{Id}^{(4)}, \Lambda^{8}\right)$ est triviale. Il suffit donc de montrer qu'il en est de même de celle de $\operatorname{Ext}_{\mathcal{P}}^{*}\left(\operatorname{Id}^{(4)}, K^{\prime}\right)$. Comme la dimension totale de l'espace vectoriel $\operatorname{Ext}_{\mathcal{P}}^{*+1}\left(\operatorname{Id}^{(4)}, S_{(7,1)^{\prime}}\right)$ est 2, et celle de $\operatorname{Ext}_{\mathcal{P}}^{*}\left(\operatorname{Id}^{(4)}, \Lambda^{8}\right)$ est 1 , la dimension totale de $\operatorname{Ext}_{\mathcal{P}}^{*}\left(\operatorname{Id}^{(4)}, K\right)$ est 1 , ce qui implique que cet espace a une structure de module de Yoneda triviale. Cela achève la preuve.

La démonstration ci-dessus est basée sur la figure 1, donnant la structure de $\Lambda^{6} \otimes \Lambda^{2}$.

Dans ce diagramme, les partitions représentées aux sommets du graphe désignent les foncteurs simples associés aux duaux des partitions; ce sont les foncteurs simples apparaissant dans la série de décomposition de $\Lambda^{2} \circ \Lambda^{6}$. Les sommets du haut correspondent aux quotients, tandis que les sommets du bas correspondent aux sous-objets. Prendre le noyau de la projection sur $S_{(7,1)^{\prime}}$ consiste à couper le graphe selon les pointillés : les deux composantes connexes qui apparaissent correspondent aux deux facteurs directs $\Lambda^{8}$ et $K^{\prime}$.

Des arguments analogues résultant de l'étude de la structure des produits tensoriels $\Lambda^{3} \otimes \Lambda^{5}$ et $\Lambda^{4} \otimes \Lambda^{4}$ via la théorie des représentations du groupe 


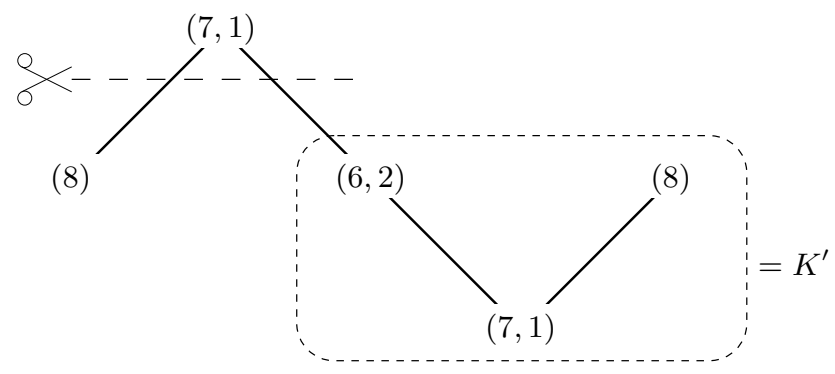

Fig. 1. Structure de $\Lambda^{6} \otimes \Lambda^{2}$

symétrique permettent de montrer de manière similaire (mais avec plus de peine) que $W_{(6,2)^{\prime}}, S_{(6,2)^{\prime}}, W_{(5,3)^{\prime}}$ et $W_{(5,3)^{\prime}}$ vérifient aussi la propriété $(\mathcal{H})$. Cela amène la question suivante :

Question 7.4. Les foncteurs simples et les foncteurs de Schur vérifientils tous la propriété $(\mathcal{H})$ ? Quels sont les foncteurs vérifiant la propriété $(\mathcal{H})$ ?

On termine cette section en montrant qu'il existe des foncteurs dans $\mathcal{P}$ n'ayant pas de "détwistés" vérifiant la propriété $(\mathcal{H})$.

Soit $\mathrm{Id}^{(2)} \rightarrow I^{0} \rightarrow I^{1} \rightarrow \ldots$ une résolution injective de $\operatorname{Id}^{(2)}$ dans $\mathcal{P}$. Soit $K$ le noyau de $I^{2} \rightarrow I^{3}$. La description de $\operatorname{Ext}_{\mathcal{P}}^{*}\left(\operatorname{Id}^{(2)}, \operatorname{Id}^{(2)}\right)$ donnée dans le théorème 1.3 permet de déterminer $\operatorname{Ext}_{\mathcal{P}}^{*}\left(\operatorname{Id}^{(2)}, K\right)$ :

$$
\operatorname{Ext}_{\mathcal{P}}^{i}\left(\operatorname{Id}^{(2)}, K\right)= \begin{cases}\mathbb{F}_{2} & \text { si } i=0,2,4 \\ 0 & \text { sinon }\end{cases}
$$

La structure de module est décrite comme suit : l'action de $e_{1}^{(1)}$ (avec la notation définie dans le théorème 1.3) est un isomorphisme de la classe de degré 2 vers la classe de degré 4 ; l'action de $e_{2}$ est un isomorphisme de la classe de degré 0 vers la classe de degré 4 . Les autres actions sont nulles. En particulier, la structure de module n'est pas triviale. Or, $K$ n'est pas égal à un foncteur twisté $L^{(1)}$, car cela impliquerait, d'après le théorème 1.7 , que sa dimension totale est paire.

\section{Références}

[1] S. Betley and T. Pirashvili, Stable K-theory as a derived functor, J. Pure Appl. Algebra 96 (1994), 245-258; appendice de T. Pirashvili.

[2] H. Cartan, Algèbre d'Eilenberg-MacLane et homotopie, Séminaire Cartan 1954/55.

[3] H. Cartan and S. Eilenberg, Homological Algebra, Princeton Univ. Press, 1956.

[4] V. Franjou, E. Friedlander, A. Scorichenko and A. Suslin, General linear and functor cohomology over finite fields, Ann. of Math. 150 (1999), 663-728.

[5] V. Franjou, J. Lannes et L. Schwartz, Autour de la cohomologie de MacLane des corps finis, Invent. Math. 115 (1994), 513-538.

[6] E. Friedlander and A. Suslin, Cohomology of finite group schemes over a field, ibid. 127 (1997), 209-270. 
[7] H.-W. Henn, J. Lannes and L. Schwartz, Analytic functors, unstable algebras and cohomology of classifying spaces, in: Algebraic Topology (Evanston, IL, 1988), Contemp. Math. 96, Amer. Math. Soc., 1989, 197-220.

[8] G. James and A. Kerber, The Representation Theory of the Symmetric Group, Encyclopedia Math. Appl. 16, Addison-Wesley, 1981.

[9] J. Jantzen, Representations of Algebraic Groups, Academic Press, 1987.

[10] M. Jibladze and T. Pirashvili, Cohomology of algebraic theories, J. Algebra 137 (1991), 253-296.

[11] T. Pirashvili, Vanishing of Ext-groups in functor category, preprint, MPI 91-19, Bonn.

[12] L. Piriou et L. Schwartz, Extensions de foncteurs simples, K-Theory 15 (1998), 269-291.

[13] L. Schwartz, Unstable Modules over the Steenrod Algebra and Sullivan's Fixed Point Set Conjecture, Univ. of Chicago Press, 1994.

[14] A. Troesch, Cohomologie de compositions de puissances symétriques, C. R. Acad. Sci. Paris Sér. I Math. 333 (2001), 509-512.

[15] - Quelques calculs de cohomologie de compositions de puissances symétriques, Comm. Algebra 30 (2002), 3351-3382.

[16] - Cohomologie de compositions de puissances symétriques en caractéristique 2, prépublication 2002-12, LAGA (Paris 13).

[17] —, Comparaison des modules d'extensions dans des catégories de foncteurs, prépublication 497, CRM, Barcelone.

[18] C. Weibel, An Introduction to Homological Algebra, Cambridge Stud. Adv. Math. 38, Cambridge Univ. Press, 1994.

LAGA, Institut Galilée

Université Paris 13

99, avenue J.-B. Clément

93430 Villetaneuse, France

E-mail: troesch@math.univ-paris13.fr

Received 30 October 2002;

in revised form 3 February 2003 